\title{
Spectrum White Space Trade in Cognitive Radio Networks
}

\author{
Gaurav S. Kasbekar and Saswati Sarkar
}

\begin{abstract}
We study price competition among primaries in a Cognitive Radio Network (CRN) with multiple primaries and secondaries located in a large region. In every slot, each primary has unused bandwidth with some probability, which may be different for different primaries. Also, there may be a random number of secondaries. A primary can lease out its unused bandwidth to a secondary in exchange for a fee. Each primary tries to attract secondaries by setting a lower price for its bandwidth than the other primaries. Radio spectrum has the distinctive feature that transmissions at neighboring locations on the same channel interfere with each other, whereas the same channel can be used at far-off locations without mutual interference. So in the above price competition scenario, each primary must jointly select a set of mutually non-interfering locations within the region (which corresponds to an independent set in the conflict graph representing the region) at which to offer bandwidth and the price at each location. In this paper, we analyze this price competition scenario as a game and seek a Nash Equilibrium (NE). For the game at a single location, we explicitly compute a NE and prove its uniqueness. Also, for the game at multiple locations, we identify a class of conflict graphs, which we refer to as mean valid graphs, such that the conflict graphs of a large number of topologies that commonly arise in practice are mean valid. We explicitly compute a $\mathrm{NE}$ in mean valid graphs and show that it is unique in the class of $\mathrm{NE}$ with symmetric independent set selection strategies of the primaries.
\end{abstract}

\section{INTRODUCTION}

The emerging cognitive radio technology [1] promises efficient usage of the available radio spectrum. In cognitive radio networks (CRNs), there are two types of spectrum users: (i) primary users who lease portions (channels or bands) of the spectrum directly from the regulator, and (ii) secondary users who lease channels from primaries and can use a channel when it is not in use by the primary. Time is slotted, and in every slot, each primary has unused bandwidth with some probability, which it would like to sell to secondaries. Now, secondaries buy bandwidth from the primaries that offer it at a low price, which results in price competition among the primaries. If a primary quotes a low price, it will attract buyers, but will earn lower profit per sale. This is a common feature of an oligopoly [7], in which multiple firms sell a common good to a pool of buyers. Price competition in an oligopoly is naturally modeled using game theory [2], and has been extensively studied in economics using, for example, the classic Bertrand game [7] and its variants.

However, a CRN has several distinguishing features, which makes the price competition very different from oligopolies encountered in economics. First, in every slot, each primary may or may not have unused bandwidth available. Second,

G. Kasbekar and S. Sarkar are with the Department of Electrical and Systems Engineering at University of Pennsylvania, Philadelphia, PA, U.S.A. Their email addresses are kgaurav@seas.upenn.edu and swati@seas.upenn.edu respectively. the number of secondaries will be random and not known apriori as each secondary may be a local spectrum provider or even a user shopping for spectrum in a futuristic scenario, e.g., users at airports, hotspots, etc. Thus, each primary who has unused bandwidth is uncertain about the number of primaries from whom it will face competition as well as the demand for bandwidth; it may only have access to imperfect information such as statistical distributions about either. A low price will result in unnecessarily low revenues in the event that very few other primaries have unused bandwidth or several secondaries are shopping for bandwidth, because even with a higher price the primary's bandwidth would have been bought, and vice versa. Third, spectrum is a commodity that allows spatial reuse: the same band can be simultaneously used at far-off locations without interference; on the other hand, simultaneous transmissions at neighboring locations on the same band interfere with each other. Thus, spatial reuse provides an opportunity to primaries to increase their profit by selling the same band to secondaries at different locations, which they can utilize subject to satisfying the interference constraints. So when multiple primaries own bandwidth in a large region, each needs to decide on a set of non-interfering locations in the region, which corresponds to an independent set in the conflict graph representing the region, at which to offer bandwidth. This is another source of strategic interaction among the primaries- each primary would like to select a maximum-sized independent set to offer bandwidth at; but if a lot of primaries offer bandwidth at the same locations, there is intense competition at those locations. So a primary would have benefited by instead offering bandwidth at a smaller independent set and charging high prices at those locations.

Pricing related issues have been extensively studied in the context of wired networks and the Internet; see [12] for an overview. Price competition among spectrum providers in wireless networks has been studied in [13], [14], [15], [16], [17], [18]. Specifically, Niyato et. al. analyze price competition among multiple primaries in CRNs [17], [18]. However, neither uncertain bandwidth availability, nor spatial reuse is modeled in any of the above papers. Also, most of these papers do not explicitly find a Nash Equilibrium (NE) (exceptions are [14], [17]). Our model incorporates both uncertain bandwidth availability and spatial reuse, which makes the problem challenging; despite this, we are able to explicitly compute a NE. Zhou et. al. [19] have designed double auction based spectrum trades in which an auctioneer chooses an allocation taking into account spatial reuse and bids. However, in the price competition model we consider, each primary independently sells bandwidth, and hence a central entity such as an auctioneer is not required. In the economics literature, the Bertrand game [7] and several of its 
variants [8], [9], [10], [11], [20] have been used to study price competition. Osborne et al [8] consider price competition in a duopoly, when the capacity of each firm is constrained. Chawla et al. [20] consider price competition in networks where each seller owns a capacity-constrained link, and decides the price for using it; the consumers choose paths they would use in the networks based on the prices declared and pay the sellers accordingly. The capacities in both cases are deterministic, whereas the availability of bandwidth is random in our model.

The closest to our work are [10], [11], which analyze price competition where each seller may be inactive with some probability, as also our prior work [22], [25], [26] in which we analyzed price competition in a CRN. The above body of work however suffers from the limitation that they either consider (i) only the symmetric model where the bandwidth availability probability of each seller is the same [10], [11], [22], [26] or (ii) primaries and secondaries located at a single location [10], [11], [25] (i.e., no spatial reuse) ${ }^{1}$. In addition, the results in [10], [11] are restricted to the case of one buyer, and [25] assumes a fixed, and apriori known number of secondaries, whereas a CRN is likely to have an unknown and random number of secondaries, which we consider in this paper. Characterizing the Nash Equilibrium (NE) in either asymmetric games (i.e., when different primaries have different bandwidth availability probabilities in our context) or in games over graphs (i.e., in presence of spatial reuse in our context) is usually quite challenging, and the combination of the above often turns out to be analytically untractable. This is the space where we seek to contribute in this paper.

We consider price competition in a CRN with multiple primaries and multiple secondaries, where each primary has available bandwidth in a slot with a certain probability, which may be different for different primaries. Also, the number of secondaries may be random and unknown to the primaries, with only their distribution being known. First, we analyze the case of primaries and secondaries in a single location (Section III). Since prices can take real values, the strategy sets of players are continuous. In addition, the utilities of the primaries are not continuous functions of their actions. Thus, classical results, including those for concave and potential games, do not establish the existence and uniqueness of $\mathrm{NE}$ in the resulting game, and there is no standard algorithm for finding a NE. Nevertheless, we are able to explicitly compute a NE and show that it is unique in the class of all NE, even allowing for player strategies that are arbitrary mixtures of continuous and discrete probability distributions (Section III).

We subsequently model the scenario where each primary owns bandwidth across multiple locations using a conflict graph in which there is an edge between each pair of mutually interfering locations (Section II-A). Each primary must simultaneously select a set of mutually non-interfering locations (independent set) at which to offer bandwidth and the prices at those locations. We focus on a class of conflict graphs that we refer to as mean valid graphs. As we show in Section IV-B, it turns out that the conflict graphs of a large number of

\footnotetext{
${ }^{1}$ In [22], the asymmetric case is considered only for a toy model with two primaries and one secondary; [25] largely focuses on a single location game, except for a limited analysis of spatial reuse in the setting of a linear conflict graph.
}

topologies that arise in practice are mean valid. We show that a mean valid graph has a unique NE in the class of NE with symmetric independent set selection strategies of the primaries (Section IV-C). Also, this NE has a simple form and the NE strategies can be explicitly computed by solving a system of equations that we provide. Finally, we prove that in the limit as the numbers of primaries and secondaries go to infinity, the NE structure exhibits interesting threshold behavior: in particular, the efficiency of this NE, which is the ratio of the aggregate revenue of all the primaries under the NE and the maximum possible aggregate revenue, changes from 1 to 0 as the average bandwidth availability increases relative to the average bandwidth demand at each location.

We defer all the proofs until the Appendix.

\section{Model And OBJective}

\section{A. Model}

Suppose there are $n \geq 2$ primaries, each of whom owns a channel throughout a large region which is a geographically well-separated or separately administered area, such as a state or a country ${ }^{2}$. The channels owned by the primaries are all orthogonal to each other. Time is divided into slots of equal duration. In every slot, each primary independently either uses its channel throughout the region to satisfy its own subscriber demand, or does not use it anywhere in the region. A typical scenario where this happens is when primaries broadcast the same signal over the entire region, e.g., if they are television broadcasters. For $i \in\{1, \ldots, n\}$, let $q_{i} \in(0,1)$ be the probability that primary $i$ does not use its channel in a slot (to satisfy its subscriber demand). Without loss of generality, we assume that:

$$
q_{1} \geq q_{2} \geq \ldots \geq q_{n}
$$

Now, the region contains smaller parts, which we refer to as locations. For example, the large region may be a state, and the locations may be towns within it.

Each secondary may be a local spectrum provider or even a user seeking to lease spectrum bands to transmit data on an on-demand basis at a location. In practice, the number of secondaries seeking to buy bandwidth may be random and unequal at different locations and also apriori unknown to the primaries, due to user mobility, varying bandwidth requirements of the secondaries, etc. Thus, the number of secondaries seeking to buy bandwidth (henceforth referred to as the number of secondaries for simplicity) at a location $v$ is $K_{v}$, where $K_{v}$ is a random variable with probability mass function (p.m.f.) $\operatorname{Pr}\left(K_{v}=k\right)=\gamma_{k}$. Also, the random variables $K_{v}$ at different nodes $v$ may be correlated. The primaries apriori know only the $\gamma_{k} \mathrm{~s}$, but not the values of $K_{v}$ for any given location $v$. We will make some technical assumptions on the p.m.f. $\left\{\gamma_{k}\right\}$ : (i) $\sum_{k=0}^{n-1} \gamma_{k}>0$ (i.e., the total number of primaries exceeds the number of secondaries with positive probability, but not necessarily probability 1 ) (ii) if $\gamma_{0}>0$, then $\gamma_{1}>0$ (if the event that no secondary requires bandwidth has positive probability, then the event that only 1 secondary requires bandwidth also has positive probability). A large class of probability mass functions, including those

\footnotetext{
${ }^{2} \mathrm{We}$ assume that all the primaries own bandwidth in the same region.
} 
generated from the most common scenario, where each local provider or user from a given pool requires bandwidth with a positive probability independent of others, satisfy both the above assumptions.

A primary who has unused bandwidth in a slot can lease it out to secondaries at a subset of the locations, provided this subset satisfies the spatial reuse constraints, which we describe next. The overall region can be represented by an undirected graph [6] $G=(V, E)$, where $V$ is the set of nodes and $E$ is the set of edges, called the conflict graph, in which each node represents a location, and there is an edge between two nodes iff transmissions at the corresponding locations interfere with each other. Note that graphs have been widely used to model ad hoc networks, wherein wireless devices are modeled as nodes in an undirected graph, with mutually interfering nodes being connected by an edge (e.g., see [23]). However, the concept of spatial reuse in our paper is more closely related to the corresponding notion in cellular networks, where cells are represented by nodes in an undirected graph, with interfering cells corresponding to neighbors in the graph [24]. Recall that an independent set [6] (I.S.) in a graph is a set of nodes such that there is no edge between any pair of nodes in the set. Now, a primary who is not using its channel must offer it at a set of mutually non-interfering locations, or equivalently, at an I.S. of nodes; otherwise secondaries ${ }^{3}$ will not be able to successfully transmit simultaneously using the bandwidth they purchase, owing to mutual interference.

A primary $i$ who offers bandwidth at an I.S. $I$, must also determine for each node $v \in I$, the access fee, $p_{i, v}$, to be charged to a secondary if the latter leases the bandwidth at node $v$. A primary incurs a cost of $c \geq 0$ per slot per node for leasing out bandwidth. This cost may arise, for example, if the secondary uses its infrastructure to access the Internet. We assume that $p_{i, v} \leq \nu$ for each primary $i$ and each node $v$, for some constant $\nu>c$. This upper bound $\nu$ may arise as follows. (1) The spectrum regulator may impose this upper bound to ensure that primaries do not excessively overprice bandwidth even when competition is limited owing to bandwidth scarcity or high demands from secondaries, or when the primaries collude. (2) Alternatively, the valuation of each secondary for 1 unit of bandwidth may be $\nu$, and no secondary will buy bandwidth at a price that exceeds its valuation. We assume that the primaries know this upper limit $\nu$.

Secondaries buy bandwidth from the primaries that offer the lowest price. More precisely, in a given slot, let $Z_{v}$ be the number of primaries who offer unused bandwidth at node $v$. Then, since there are $K_{v}$ secondaries at the node, the bandwidth of the $\min \left(Z_{v}, K_{v}\right)$ primaries that offer the lowest prices is bought (ties are resolved at random) at the node.

If primary $i$ has unused bandwidth, then the utility or payoff of primary $i$ is defined to be its net revenue ${ }^{4}$. Also, we consider an additive utility function, which is natural in the context of monetary profits. So the utility of a primary $i$ who

\footnotetext{
${ }^{3}$ Note that secondaries usually purchase bandwidth for communication (and not television broadcasts). Thus, two secondaries can not use the same band simultaneously at interfering locations.

${ }^{4}$ If instead, the utility were defined to be primary $i$ 's net revenue, unconditional on whether it has unused bandwidth or not, then the expected utilities of primary $i$ in the game analysis would all be scaled by $q_{i}$.
}

offers bandwidth at an I.S. $I$ and sets a price of $p_{i, v}$ at node $v \in I$ is given by $\sum\left(p_{i, v}-c\right)$, where the summation is over the nodes $v \in I$ at which primary $i$ 's bandwidth is bought. (The utility is 0 if bandwidth is not bought at any node).

Thus, each primary must jointly select an I.S. at which to offer bandwidth, and the prices to set at the nodes in it. Both the I.S. and price selection may be random. Thus, a strategy, say $\psi_{i}$, of a primary $i$ provides a probability mass function (p.m.f.) for selection among the I.S., and the price distribution it uses at each node (both selections contingent on having unused bandwidth). Note that we allow a primary to use different (and arbitrary) price distributions for different nodes (and therefore allow, but do not require, the selection of different prices at different nodes), and arbitrary p.m.f. (i.e., discrete distributions) for selection among the different I.S. The vector $\left(\psi_{1}, \ldots, \psi_{n}\right)$ of strategies of the primaries is called a strategy profile [7]. Let $\psi_{-i}=\left(\psi_{1}, \ldots, \psi_{i-1}, \psi_{i+1}, \ldots, \psi_{n}\right)$ denote the vector of strategies of primaries other than $i$. Let $E\left\{u_{i}\left(\psi_{i}, \psi_{-i}\right)\right\}$ denote the expected utility of primary $i$ when it adopts strategy $\psi_{i}$ and the other primaries adopt $\psi_{-i}$.

\section{B. Nash Equilibrium}

We use the Nash Equilibrium solution concept, which has been extensively used in game theory in general and wireless network applications in particular to predict the outcome of a game.

Definition 1 (Nash Equilibrium (NE)): A Nash equilibrium (NE) is a strategy profile such that no player can improve its expected utility by unilaterally deviating from its strategy [7]. Thus, $\left(\psi_{1}^{*}, \ldots, \psi_{n}^{*}\right)$ is a NE if for each primary $i$ :

$$
E\left\{u_{i}\left(\psi_{i}^{*}, \psi_{-i}^{*}\right)\right\} \geq E\left\{u_{i}\left(\widetilde{\psi}_{i}, \psi_{-i}^{*}\right)\right\}, \forall \widetilde{\psi}_{i}
$$

Equation (2) says that when players other than $i$ play $\psi_{-i}^{*}$, $\psi_{i}^{*}$ maximizes $i$ 's expected utility; $\psi_{i}^{*}$ is said to be its best response [7] to $\psi_{-i}^{*}$.

Note that the existence of a NE is not apriori clear even in the simplest possible setting of a single location, far less the uniqueness and characterization of NE strategy profiles. This is because the prices can take real values and hence the strategy sets of players are not finite. In addition, the utilities of the primaries are not continuous functions of their actions. For example, consider the game in which there is a single location $v, n=2$ primaries and $K_{v}=1$ secondary with probability 1 . If primary 1 has unused bandwidth, its expected utility is

$$
\begin{cases}p_{1, v}-c & \text { if } p_{1, v}<p_{2, v} \\ \left(p_{1, v}-c\right) / 2 & \text { if } p_{1, v}=p_{2, v} \\ \left(1-q_{2}\right)\left(p_{1, v}-c\right) & \text { if } p_{1, v}>p_{2, v} .\end{cases}
$$

which is a discontinuous function of the prices. Thus, classical results, including those for concave and potential games, do not establish the existence of $\mathrm{NE}$ in the resulting game, and there is no standard algorithm for finding a NE.

\section{Single LOCATION}

In this section, we analyze price competition when all the primaries and secondaries are present in a single location. Let the (random) number of secondaries at this location be denoted as $K$. Since there is only one location, there are no spatial reuse constraints, and the strategy of a primary $i$ is 
a distribution function (d.f.) ${ }^{5} \psi_{i}($.$) , which it uses to select$ the price $p_{i}$. For convenience, we define the pseudo-price of primary $i \in\{1, \ldots, n\}, p_{i}^{\prime}$, as the price it selects if it has unused bandwidth and $p_{i}^{\prime}=\nu+1$ otherwise ${ }^{6}$. Also, let $\phi_{i}($. be the d.f. of $p_{i}^{\prime}$. For $c \leq x \leq \nu, p_{i}^{\prime} \leq x$ for a primary $i$ iff it has unused bandwidth and sets a price $p_{i} \leq x$. So $\phi_{i}(x)=q_{i} P\left(p_{i} \leq x\right)=q_{i} \psi_{i}(x)$. Thus, $\psi_{i}($.$) and \phi_{i}($. differ only by a constant factor on $[c, \nu]$ and we use them interchangeably wherever applicable.

\section{A. Necessary Conditions for a NE}

Consider a NE under which the d.f. of the price (respectively, pseudo-price) of primary $i$ is $\psi_{i}($.$) (respectively, \phi_{i}($.$) ).$ In Theorem 1 below, we show that the NE strategies must have a particular structure. Before stating Theorem 1, we describe some basic properties of the NE strategies.

Property 1: $\phi_{2}(),. \ldots, \phi_{n}($.$) are continuous on [c, \nu] . \phi_{1}($. is continuous at every $x \in[c, \nu)$, has a jump ${ }^{7}$ of size $q_{1}-q_{2}$ at $\nu$ if $q_{1}>q_{2}$ and is continuous at $\nu$ if $q_{1}=q_{2}$.

Thus, there does not exist a pure strategy NE (one in which every primary selects a single price with probability (w.p.) 1 ).

Now, let $u_{i, \max }$ be the expected payoff that primary $i$ gets in the NE and $L_{i}$ be the lower endpoint of the support set ${ }^{8}$ of $\psi_{i}($.$) , i.e.:$

$$
L_{i}=\inf \left\{x: \psi_{i}(x)>0\right\} .
$$

Also, let $w_{i}$ be the probability of the event that at least $K$ primaries among $\{1, \ldots, n\} \backslash i$ have unused bandwidth. Let $r$ be the probability that $K \geq 1$. Note that $r=1-\gamma_{0}$, and $w_{i}$ can be easily computed using the p.m.f $\left\{\gamma_{k}\right\}$ and the fact that each primary $j$ independently has unused bandwidth w.p. $q_{j}$.

Property 2: $L_{1}=\ldots L_{n}=\tilde{p}$, where $\tilde{p}=c+\frac{(\nu-c)\left(1-w_{1}\right)}{r}$. Also, $u_{i, \max }=(\tilde{p}-c) r, i=1, \ldots, n$.

Thus, the lower endpoints of the support sets of the d.f.s $\psi_{1}(),. \ldots, \psi_{n}($.$) of all the primaries are the same.$

Theorem 1: The following are necessary conditions for strategies $\phi_{1}(),. \ldots, \phi_{n}($.$) to constitute a NE:$

1) $\phi_{1}(),. \ldots, \phi_{n}($.$) satisfy Property 1$ and Property 2.

2) There exist numbers $R_{j}, j=1, \ldots, n+1$, and a function $\{\phi(x): x \in[\tilde{p}, \nu)\}$ such that

$$
\begin{gathered}
\tilde{p}=R_{n+1}<R_{n} \leq R_{n-1} \leq \ldots \leq R_{1} \leq \nu \\
\phi_{1}(x)=\ldots=\phi_{j}(x)=\phi(x), \tilde{p} \leq x<R_{j}, j \in\{1, \ldots, n\}, \\
\quad \text { and } \phi_{j}\left(R_{j}\right)=q_{j}, j=1, \ldots, n .
\end{gathered}
$$

Also, every point in $\left[\tilde{p}, R_{j}\right)$ is a best response for primary $j$ and it plays every sub-interval in $\left[\tilde{p}, R_{j}\right)$ with positive probability. Finally, $R_{1}=R_{2}=\nu$.

Theorem 1 says that all $n$ primaries play prices in the range $\left[\tilde{p}, R_{n}\right)$, the d.f. $\phi_{n}($.$) of primary n$ stops increasing at $R_{n}$, the remaining primaries $1, \ldots, n-1$ also play prices in the range $\left[R_{n}, R_{n-1}\right)$, the d.f. $\phi_{n-1}($.$) of primary n-1$ stops increasing

\footnotetext{
${ }^{5}$ Recall that the d.f. of a random variable $X$ is the function $f(x)=P(X \leq$ $x), x \in R$, where $R$ denotes the set of real numbers.

${ }^{6}$ The choice $\nu+1$ is arbitrary. Any other choice greater than $\nu$ also works.

${ }^{7} \mathrm{~A}$ d.f. $f(x)$ is said to have a jump (discontinuity) of size $b>0$ at $x=a$ if $f(a)-f(a-)=b$, where $f(a-)=\lim _{x \uparrow a} f(x)$.

${ }^{8}$ The support set of a d.f. is the smallest closed set such that its complement has probability zero under the d.f.
}

at $R_{n-1}$, and so on. Also, primary 1's d.f. $\phi_{1}($.$) has a jump of$ height $q_{1}-q_{2}$ at $\nu$ if $q_{1}>q_{2}$. Fig. 1 illustrates the structure.

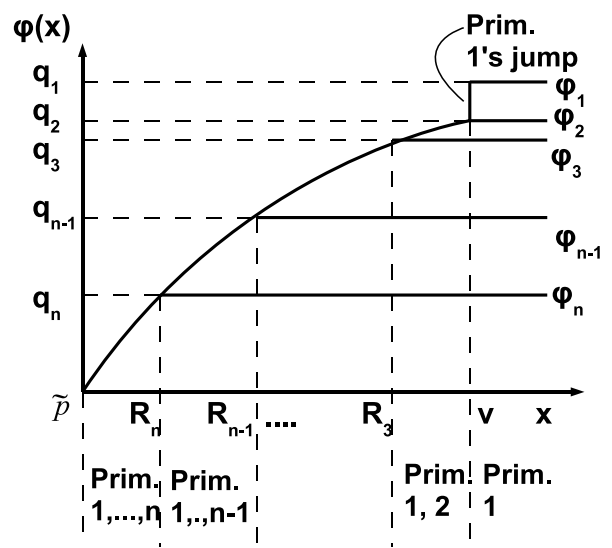

Fig. 1. The figure shows the structure of a NE described in Theorem 1. The horizontal axis shows prices in the range $x \in[\tilde{p}, \nu]$ and the vertical axis shows the functions $\phi($.$) and \phi_{1}(),. \ldots, \phi_{n}($.$) .$

\section{B. Explicit Computation, Uniqueness and Sufficiency}

By Theorem 1, for each $i \in\{1, \ldots, n\}$ :

$$
\phi_{i}(x)= \begin{cases}\phi(x), & \tilde{p} \leq x<R_{i} \\ q_{i}, & x \geq R_{i}\end{cases}
$$

So the candidate NE strategies $\phi_{1}(),. \ldots, \phi_{n}($.$) are completely$ determined once $\tilde{p}, R_{1}, \ldots, R_{n}$ and the function $\phi($.$) are$ specified. Also, Property 2 provides the value of $\tilde{p}$, and $R_{1}=R_{2}=\nu$ by Theorem 1 . First, we will show that there also exist unique $R_{3}, \ldots, R_{n}$ and $\phi($.$) satisfying (4),$ (5), and (6) and will compute them. Then, we will show that the resulting strategies given by (7) indeed constitute a NE (sufficiency).

Let $p_{-i}^{\prime}$ be the $K$ 'th smallest pseudo-price out of the pseudo-prices, $\left\{p_{l}^{\prime}: l \in\{1, \ldots, n\}, l \neq i\right\}$, of the primaries other than $i$ (with $p_{-i}^{\prime}=0$ if $K=0$ and $p_{-i}^{\prime}=\nu+2$ if $K>n-1)$. Also, let $F_{-i}(x)$ denote the d.f. of $p_{-i}^{\prime}$. Since there are $K$ secondaries, if primary 1 has unused bandwidth and sets $p_{1}=x \in[\tilde{p}, \nu)$, its bandwidth is bought iff ${ }^{9} p_{-1}^{\prime}>x$, which happens w.p. $1-F_{-1}(x)$. Note that primary 1's payoff is $(x-c)$ if its bandwidth is bought and 0 otherwise. So, letting $E\left\{u_{i}\left(x, \psi_{-i}\right)\right\}$ denote the expected payoff of primary $i$ if it sets a price $x$ and the other primaries use the strategy profile $\psi_{-i}$, we have:

$E\left\{u_{1}\left(x, \psi_{-1}\right)\right\}=(x-c)\left(1-F_{-1}(x)\right)=(\tilde{p}-c) r, x \in[\tilde{p}, \nu)$

where the second equality follows from the facts that each $x \in[\tilde{p}, \nu)$ is a best response for primary 1 by Theorem 1 , and $u_{1, \max }=(\tilde{p}-c) r$ by Property 2 . By (8), we get:

$$
\begin{gathered}
F_{-1}(x)=g(x), x \in[\tilde{p}, \nu) . \\
\text { where, } g(x)=\frac{x-c-(\tilde{p}-c) r}{x-c}, x \in[\tilde{p}, \nu) .
\end{gathered}
$$

Next, we calculate $R_{i}, i=3, \ldots, n$ and $\phi($.$) using (9).$

\footnotetext{
${ }^{9}$ By Property 1 , no primary has a jump at any $x \in[\tilde{p}, \nu)$. So $P\left(p_{-1}^{\prime}=\right.$ $x)=0$.
} 
1) Computation of $R_{i}, i=3, \ldots, n$ : For $0 \leq y \leq 1$, let $f_{i}(y)$ be the probability of $K$ or more successes out of $n-$ 1 independent Bernoulli events, $(i-1)$ of which have the same success probability $y$ and the remaining $(n-i)$ have success probabilities $q_{i+1}, \ldots, q_{n}$. An expression for $f_{i}(y)$ can be easily computed.

Now, to compute $R_{i}, i \in\{3, \ldots, n\}$, we note that by (7) and (4), $\phi_{j}\left(R_{i}\right)=q_{i}, j=2, \ldots, i$, and $\phi_{j}\left(R_{i}\right)=q_{j}, j=$ $i+1, \ldots, n$. So from the preceding paragraph, with the events $\left\{p_{j}^{\prime} \leq R_{i}\right\}, j=2, \ldots, n$ as the $n-1$ Bernoulli events, and by the definition of $F_{-1}($.$) , we get:$

$$
F_{-1}\left(R_{i}\right)=f_{i}\left(q_{i}\right) .
$$

By (9) and (11):

$$
g\left(R_{i}\right)=f_{i}\left(q_{i}\right) .
$$

By (10) and (12), $R_{i}$ is unique and is given by:

$$
R_{i}=c+\frac{(\tilde{p}-c) r}{1-f_{i}\left(q_{i}\right)} .
$$

2) Computation of $\phi($.$) : Now we compute the function$ $\{\phi():. x \in[\tilde{p}, \nu)\}$ by separately computing it for each interval $\left[R_{i+1}, R_{i}\right), i \in\{2, \ldots, n\}$. If $R_{i+1}=R_{i}$, then note that the interval $\left[R_{i+1}, R_{i}\right)$ is empty. Now suppose $R_{i+1}<R_{i}$. For $x \in\left[R_{i+1}, R_{i}\right)$, by (7) and (4):

$$
\begin{gathered}
\phi_{j}(x)=q_{j}, j=i+1, \ldots, n \\
\text { and } \phi_{1}(x)=\ldots=\phi_{i}(x)=\phi(x) .
\end{gathered}
$$

By definition of the function $f_{i}($.$) , with the events \left\{p_{j}^{\prime} \leq\right.$ $x\}, j=2, \ldots, n$ as the $n-1$ Bernoulli events, by definition of $F_{-1}(x)$ and using $P\left\{p_{j}^{\prime} \leq x\right\}=\phi_{j}(x)$, (14) and (15):

$$
F_{-1}(x)=f_{i}(\phi(x)), R_{i+1} \leq x<R_{i} .
$$

By (9) and (16):

$$
f_{i}(\phi(x))=g(x), R_{i+1} \leq x<R_{i} .
$$

Lemma 1: For each $x$, (17) has a unique solution $\phi(x)$. The function $\phi($.$) is strictly increasing and continuous on [\tilde{p}, \nu)$. For $i \in\{2, \ldots, n\}, \phi\left(R_{i}\right)=q_{i}$. Also, $\phi(\tilde{p})=0$.

Thus, there is a unique function $\phi($.$) , and by (7), unique$ $\phi_{i}(),. i=1, \ldots, n$ that satisfy the conditions in Theorem 1.

3) Sufficiency:

Theorem 2: The pseudo-price d.f.s $\phi_{i}(),. i=1, \ldots, n$ in (7), with $R_{1}=R_{2}=\nu, R_{i}, i=3, \ldots, n$ given by (13), and $\phi($.$) being the solution of (17), constitute the unique NE.$ The corresponding price d.f.s are $\psi_{i}(x)=\frac{1}{q_{i}} \phi_{i}(x), x \in[c, \nu]$, $i=1, \ldots, n$.

Thus, in the price competition game at a single location, there is a unique NE that can be computed explicitly. This $N E$ fetches equal expected payoffs for each primary, which by Property 2 is given by:

$$
(\tilde{p}-c) r=(\nu-c)\left(1-w_{1}\right) .
$$

\section{Multiple Locations}

We now study the existence, computation and uniqueness of NE in the presence of spatial reuse. Recall that a strategy of a primary now consists of a p.m.f. over I.S. and price distributions at individual nodes. Our first observation is that in general, there may be multiple $\mathrm{NE}$ in this case. For example, consider the simple setup with two nodes $v_{1}$ and $v_{2}$ connected by an edge, two primaries and one secondary with probability 1 at each node. It can be easily verified that both of the following strategy profiles constitute NEs: primary 1 offers bandwidth at node $v_{1}$ (respectively, $v_{2}$ ) if it has unused bandwidth and primary 2 at node $v_{2}$ (respectively, $v_{1}$ ) if it has unused bandwidth, and both primaries set the maximum possible price of $\nu$. The results in games with multiple locations may therefore fundamentally differ from those for a single location.

Note that obtaining the structure of NE in games over graphs is usually extremely challenging. As a result, in many problems of practical importance (e.g., base station deployment games [21]), such characterizations have been done only in small graphs with a few nodes. In spite of this, we will establish the existence of a NE and explicitly compute it for a fairly general class of graphs that we refer to as mean valid graphs. In addition, we will also prove its uniqueness in the class of NEs in which all primaries choose the I.S. they would offer bandwidth at with identical probability mass functions.

\section{A. A Separation Result}

We start by providing a separation framework from which the price distributions at individual nodes follow once the I.S. selection p.m.f.s are determined. Let $\mathscr{I}$ be the set of all I.S. in $G$. For convenience, we assume that the empty I.S. $I_{\emptyset} \in \mathscr{I}$ and we allow a primary to offer bandwidth at $I_{\emptyset}$, i.e. to not offer bandwidth at any node, with some probability. Consider a NE under which, if primary $i$ has unused bandwidth, it selects I.S. $I \in \mathscr{I}$ w.p. $\beta_{i}(I)$, where $\sum_{I \in \mathscr{I}} \beta_{i}(I)=1$. The probability, say $\alpha_{v}^{i}$, with which primary $i$ offers bandwidth at a node $v \in V$ equals the sum of the probabilities associated with all the I.S. that contain the node:

$$
\alpha_{v}^{i}=\sum_{I \in \mathscr{I}: v \in I} \beta_{i}(I) .
$$

Now, considering that primary $i$ has unused bandwidth w.p. $q_{i}$, it offers it at node $v$ w.p. $q_{i} \alpha_{v}^{i}$. The price selection problem at each node $v$ is now equivalent to that for the single location case, the difference being that primary $i$ offers unused bandwidth w.p. $q_{i} \alpha_{v}^{i}$, instead of $q_{i}$, at node $v$. Thus:

Lemma 2: Suppose under a NE primary $i \in\{1, \ldots, n\}$ selects node $v$ w.p. $\alpha_{v}^{i}$ if it has unused bandwidth. Then under that NE the price distribution of primary $i$ at node $v$ is the d.f. $\psi_{i}($.$) in Section III, with q_{1} \alpha_{v}^{1}, \ldots, q_{n} \alpha_{v}^{n}$ in place of $q_{1}, \ldots, q_{n}$ respectively all through.

Thus, the strategy profile of the primaries in an NE is completely specified once the I.S. selection p.m.f.s $\left\{\beta_{i}(I)\right.$ : $I \in \mathscr{I}, i \in\{1, \ldots, n\}\}$ (which will in turn provide the $\alpha_{v}^{i} \mathrm{~s}$ via (19)) are obtained. 


\section{B. Mean Valid Graphs}

We now introduce mean valid graphs, which model the conflict graphs of several topologies that commonly arise in practice. In the next section, we show that these graphs have a $\mathrm{NE}$, which can be explicitly computed and has a simple form; this NE will also turn out to be unique in a large class of strategy profiles.

\section{1) Definition:}

Definition 2 (Valid Distribution): An assignment $\left\{\alpha_{v}: v \in\right.$ $V\}$ of probabilities to the nodes is said to be a valid distribution if there exists a probability distribution $\{\beta(I): I \in \mathscr{I}\}$ such that for each $v \in V, \alpha_{v}=\sum_{I \in \mathscr{I}: v \in I} \beta(I)$.

Definition 3 (Mean Valid Graph): We refer to a graph $G=$ $(V, E)$ as mean valid if:

1) Its vertex set can be partitioned into $d$ disjoint maximal ${ }^{10}$ I.S. for some integer $d \geq 2: V=I_{1} \cup I_{2} \cup \ldots \cup I_{d}$, where $I_{j}, j \in\{1, \ldots, d\}$, is a maximal I.S. and $I_{j} \cap I_{m}=\emptyset, j \neq m$.

Let $\left|I_{j}\right|=M_{j}, I_{j}=\left\{a_{j, l}: l=1, \ldots, M_{j}\right\}$ and:

$$
M_{1} \geq M_{2} \geq \ldots \geq M_{d} \text {. }
$$

2) For every valid distribution ${ }^{11}$ in which a primary who has unused bandwidth offers it at node $a_{j, l}$ w.p. $\alpha_{j, l}$, $j=1, \ldots, d, l=1, \ldots, M_{j}$,

$$
\sum_{j=1}^{d} \bar{\alpha}_{j} \leq 1, \text { where } \bar{\alpha}_{j}=\frac{\sum_{l=1}^{M_{j}} \alpha_{j, l}}{M_{j}}, j \in\{1, \ldots, d\} .
$$

The first condition in Definition 3 says that $G$ is a $d$ partite graph ${ }^{12}$ and has the additional property that each of $I_{1}, \ldots, I_{d}$ is a maximal I.S.. Next, let $\left\{\alpha_{j, l}: j=1, \ldots, d ; l=\right.$ $\left.1, \ldots, M_{j}\right\}$ be an arbitrary valid distribution. Consider the distribution $\alpha_{j, l}^{\prime}=\bar{\alpha}_{j}$, with $\bar{\alpha}_{j}$ as in (21), i.e. for each $j$ and $l=1, \ldots, M_{j}, \alpha_{j, l}^{\prime}$ is set equal to the mean of $\alpha_{j, m}, m=1, \ldots, M_{j}$. If $(21)$ is true, then this distribution of means is a valid distribution because it corresponds to the I.S. distribution $\left\{\beta\left(I_{j}\right)=\bar{\alpha}_{j}, j=1, \ldots, d ; \beta\left(I_{\emptyset}\right)=\right.$ $\left.1-\sum_{j=1}^{d} \bar{\alpha}_{j} ; \beta(I)=0, I \neq I_{1}, \ldots, I_{d}, I_{\emptyset}\right\}$. Thus, Condition 2 in Definition 3 says that in $G$, the distribution of means corresponding to every valid distribution is valid- a fact that we extensively use in the proofs of the characterization of a NE in Section IV-C.

2) Examples: Technical as Definition 3 may seem, it turns out that several conflict graphs that commonly arise in practice are mean valid. For example, consider the following graphs:

1) Let $\mathcal{G}_{m}$ denote a graph that is a linear arrangement of $m \geq 2$ nodes as shown in part (a) of Fig. 2, with an edge between each pair of adjacent nodes. As an example, this would be the conflict graph for locations along a highway or a row of roadside shops.

2) We consider two types of $m \times m$ grid graphs, denoted by $\mathcal{G}_{m, m}$ (see part (b) of Fig. 2) and $\mathcal{H}_{m, m}$ (see part (a) of

\footnotetext{
${ }^{10}$ Recall that an I.S. $I$ is said to be maximal if for each node $v \notin I, I \cup\{v\}$ is not an I.S. [6].

${ }^{11}$ Note that we write $\alpha_{j, l}$ in place of $\alpha_{a_{j, l}}$ to simplify the notation.

${ }^{12}$ Recall that a graph $G=(V, E)$ is said to be $d$-partite if $V$ can be partitioned into $d$ disjoint I.S. $I_{1}, \ldots, I_{d}$ [6]. For example, when $d=2, G$ is a bipartite graph.
}

Fig. 3). In both these graphs, $m^{2}$ nodes (locations) are arranged in a square grid. In $\mathcal{G}_{m, m}$, there is an edge only between each pair of adjacent nodes in the same row or column. In $\mathcal{H}_{m, m}$, in addition to these edges, there are also edges between nodes that are neighbors along a diagonal as shown in part (a) of Fig. 3. For example, $\mathcal{G}_{m, m}$ or $\mathcal{H}_{m, m}$ may represent a shopping complex, with the nodes corresponding to the locations of shops with WiFi Access Points (AP) for Internet access. Depending on the proximity of the shops to each other and the transmission ranges of the APs, the conflict graph could be $\mathcal{G}_{m, m}$ or $\mathcal{H}_{m, m}$. $\mathcal{H}_{m, m}$ is also the conflict graph of a cellular network with square cells as shown in part (b) of Fig. 3.

3) Let $\mathcal{T}_{m, m, m}$ be a three-dimensional grid graph (see Fig. 4), which may, for example, be the conflict graph for offices in a corporate building or rooms in a hotel.

4) The conflict graph (Fig. 6) of a cellular network with hexagonal cells (Fig. 5).

5) Consider a clique ${ }^{13}$ of size $e$, where $e \geq 1$ is any integer. This is the conflict graph for any set of $e$ locations that are close to each other.

All of the above are mean valid graphs:

Theorem 3: The following graphs are mean valid, with $d$, the number of disjoint maximal I.S., indicated in each case:

1) a clique of size $e \geq 1(d=e)$,

2) a line graph $\mathcal{G}_{m}(d=2)$,

3) a two-dimensional grid graph $\mathcal{G}_{m, m}(d=2)$,

4) a two-dimensional grid graph $\mathcal{H}_{m, m}(d=4)$,

5) a three-dimensional grid graph $\mathcal{T}_{m, m, m}(d=8)$.

6) a cellular network with hexagonal cells $(d=3)^{14}$.

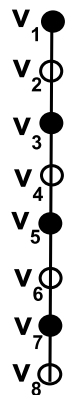

(a)

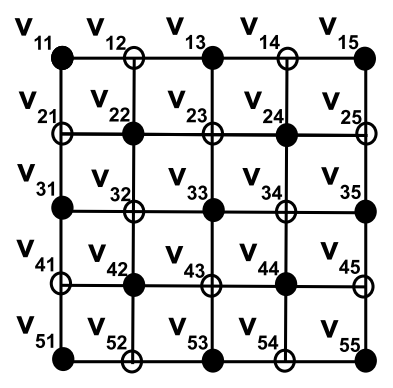

(b)
Fig. 2. Part (a) shows a linear graph, $\mathcal{G}_{m}$, with $m=8$ and part (b) shows a grid graph, $\mathcal{G}_{m, m}$, with $m=5$. Both graphs are mean valid with $d=2$ and $I_{1}$ and $I_{2}$ being disjoint maximal I.S. (in the notation of Definition 3), where the darkened and un-darkened nodes constitute $I_{1}$ and $I_{2}$ respectively.

\section{Existence and computation of a NE in Mean Valid Graphs}

Let $G$ be a mean valid graph with $d$ disjoint maximal I.S. $I_{1}, \ldots, I_{d}$. We start by considering a class of simple strategy profiles. Every primary selects I.S. $I_{j}$ with probability $t_{j}$ where

\footnotetext{
${ }^{13}$ Recall that a clique or a complete graph of size $e$ is a graph with $e$ nodes and an edge between every pair of nodes [6]

${ }^{14}$ This holds under the following assumption that eliminates problems arising due to boundary effects: There are an even number of rows of nodes, each containing $3 \delta$ nodes, for some integer $\delta \geq 1$.
} 


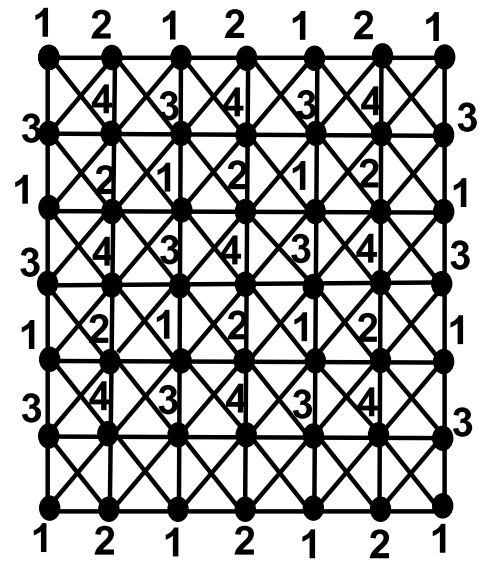

(a)

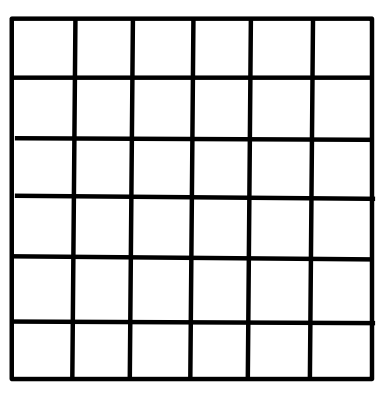

(b)
Fig. 3. Part (a) shows a grid graph $\mathcal{H}_{m, m}$ with $m=7$. It is mean valid with $d=4$ and the disjoint maximal I.S. $I_{1}, \ldots, I_{4}$ (in the notation of Definition 3), where the nodes labelled $j, j \in\{1,2,3,4\}$, constitute I.S $I_{j}$. Part (b) shows a tiling of a plane with squares, e.g. cells in a cellular network. Transmissions at neighboring cells interfere with each other. The corresponding conflict graph is $\mathcal{H}_{6,6}$.

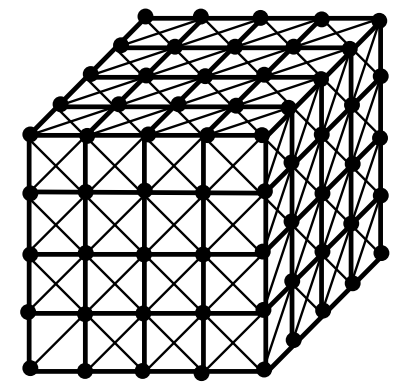

(a)

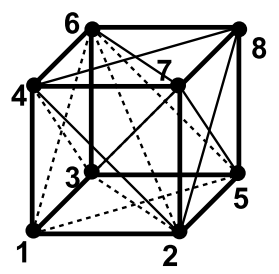

(b)
Fig. 4. Part (a) shows a three-dimensional grid graph $\mathcal{T}_{m, m, m}$ for $m=5$. It consists of periodic repetitions of the graph shown in part (b). $\mathcal{T}_{m, m, m}$ is mean valid with $d=8$ and disjoint maximal I.S. $I_{1}, \ldots, I_{8}$ (in the notation of Definition 3). In part (b), the node labels show the I.S. the nodes are in, i.e. a node with the label $j$ is part of the I.S. $I_{j}, j \in\{1, \ldots, 8\}$.

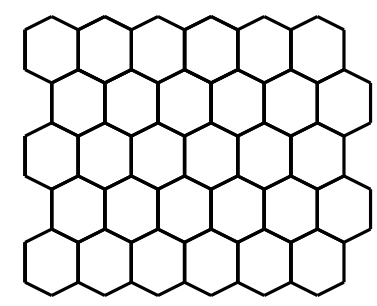

Fig. 5. The figure shows a tiling of a plane with hexagons, e.g. cells in a cellular network. Transmissions at neighboring cells interfere with each other.

$\left\{t_{j}: j=1, \ldots, d\right\}$ represents a p.m.f., i.e, $\sum_{j=1}^{d} t_{j}=1$ and $t_{j} \geq 0$ for each $j$. Interestingly enough, it turns out that a NE strategy profile belongs in this class, and furthermore, the corresponding p.m.f $\left\{t_{j}: j=1, \ldots, d\right\}$ constitutes the unique solution of a set of equations that we provide, and can

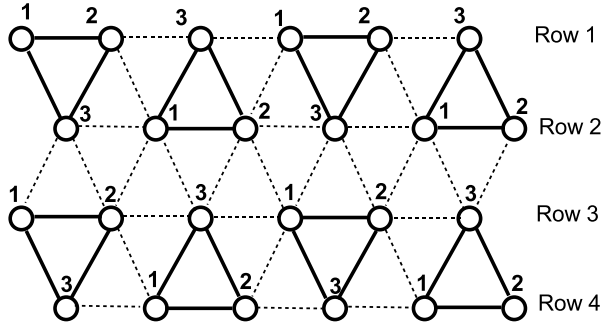

Fig. 6. The figure shows the conflict graph of a hexagonal tiling of a plane, which is mean valid with $d=3$. Both the solid and dotted edges are part of the graph. The nodes labelled $j, j \in\{1,2,3\}$, are in I.S. $I_{j}$. There are four rows of nodes. The figure also shows the construction of the graph from cliques of size 3 each, shown by the solid edges. The dotted edges are added later. Note that no edge is between two nodes in the same I.S., so the hypothesis of Lemma 17 is satisfied.

therefore be explicitly computed by solving them.

We first evaluate the expected payoff of a primary under an $\mathrm{NE}$ in the above class. We introduce some notations towards that end. Since primary $i$ has unused bandwidth w.p. $q_{i}$ and offers it at node $v \in I_{j}$ w.p. $t_{j}$, it offers bandwidth at node $v \in I_{j}$ w.p. $q_{i} t_{j}$. Analogous to the $w_{j}$ s that we introduced in Section III-A, we introduce $w_{i}\left(t_{j}\right)$ that represents the probability that $K_{v}$ or more out of primaries $\{1, \ldots, n\} \backslash i$ offer bandwidth at a given node $v \in I_{j}$ under the above I.S. p.m.f. $\left\{t_{j}: j=1, \ldots, d\right\}$. Under this p.m.f, by Lemma 2, and similar to (18) in the single location case, the primaries choose the price at each node in $I_{j}$ as per the single-node NE strategy with $q_{1} t_{j}, \ldots, q_{n} t_{j}$ in place of $q_{1}, \ldots, q_{n}$ respectively throughout, and each primary obtains an expected payoff of $W\left(t_{j}\right)$ at that node, where

$$
W(x)=\left(1-w_{1}(x)\right)(\nu-c) .
$$

Now, for simplicity, we normalize $\nu-c=1$. Then:

$$
W(x)=\left(1-w_{1}(x)\right) .
$$

Since I.S. $I_{j}$ has $M_{j}$ nodes, each primary receives a total expected payoff of $M_{j} W\left(t_{j}\right)$ if it chooses $I_{j}$.

We now state the main result of this section, which establishes the existence of a NE and also shows how it can be explicitly computed.

Theorem 4: In a mean valid graph, the following strategy profile constitutes a NE: each primary who has unused bandwidth selects I.S. $I_{j}, j \in\{1, \ldots, d\}$, w.p. $t_{j}$, where $\left(t_{1}, \ldots, t_{d}\right)$ is the unique distribution satisfying the following conditions. There exists an integer $d^{\prime}$ such that $1 \leq d^{\prime} \leq d$ and $^{15}$

$$
\begin{gathered}
t_{j}=0 \text { if } j>d^{\prime}, \text { and } \\
M_{1} W\left(t_{1}\right)=\ldots=M_{d^{\prime}} W\left(t_{d^{\prime}}\right)>M_{d^{\prime}+1} r .
\end{gathered}
$$

Also, $t_{1} \geq t_{2} \ldots \geq t_{d}$.

We first explain the result: (23) states that under the above NE, each primary selects with positive probability only some or all I.S. out of the I.S. $I_{1}, I_{2}, \ldots, I_{d}$. Since the total number of I.S. is exponential in the number of nodes in most graphs, it is surprising that an NE exists in which primaries offer

\footnotetext{
${ }^{15}$ For notational simplicity, let $M_{j}=0$ if $j>d$.
} 
bandwidth at only a small number of I.S. with positive probability. In addition, note that among $I_{1}, \ldots, I_{d}$, primaries do not select $I_{d^{\prime}+1}, \ldots I_{d}$. Recall that by (20), $I_{1}, \ldots, I_{d}$ are in decreasing order of size. So primaries do not choose I.S. smaller than a certain size (out of $I_{1}, \ldots, I_{d}$ ). Similarly, the fact that $t_{1} \geq t_{2} \ldots \geq t_{d}$ is consistent with the intuition that primaries offer bandwidth at the larger I.S. with a larger probability. Next, since $\nu-c=1$ and at each location, there exists at least one secondary w.p. $r$, whenever a primary offers bandwidth at a location, its expected payoff at that location is $r$ or less. Thus, by (20), if it would have selected an I.S. in $I_{d^{\prime}+1}, \ldots I_{d}$, it would have earned a payoff of at most $M_{d^{\prime}+1} r$. As discussed above, a primary earns an expected payoff of $M_{j} W\left(t_{j}\right)$ if it selects $I_{j}$. Thus, (24) states that a primary earns equal expected payoffs by choosing I.S. in $I_{1}, I_{2}, \ldots, I_{d^{\prime}}$ and this payoff exceeds the maximum payoff it could have earned by selecting an I.S. in $I_{d^{\prime}+1}, \ldots I_{d^{-}}$hence it never opts for the latter choice. Interestingly, although different primaries have different bandwidth availability probabilities, there exists at least one NE where all use the same I.S. selection p.m.f. They will however use different price distributions at the same node: primary $i$ selects the d.f. $\psi_{i}($.$) in Section III, with$ $q_{1} t_{j}, \ldots, q_{n} t_{j}$ in place of $q_{1}, \ldots, q_{n}$ throughout at each node in $I_{j}$ (Lemma 2).

The above theorem implies that every mean valid graph has a NE, which can be explicitly computed by solving the system of equations (23) and (24). Note that this is a system of nonlinear equations in the variables $t_{1}, \ldots, t_{d}$ and $d^{\prime}$. It can be solved using a standard solver for non-linear equations (e.g., fsolve in Matlab) in combination with a search procedure to find $d^{\prime}$. We now illustrate this NE using an example.

Example: Suppose there are $n=2$ primaries with probabilities of having unused bandwidth $q_{1}$ and $q_{2}$, where $q_{1} \geq q_{2}$, and $K_{v}=1$ secondary w.p. 1 at every node $v$. Consider a grid graph $\mathcal{H}_{m, m}$, which was introduced in Section IV-B2, with $m=7$ (see part (a) of Fig. 3). By part 4 of Theorem 3, this is a mean valid graph and, in the notation of Definition 3, $d=4$, the I.S. $I_{1}, I_{2}, I_{3}$ and $I_{4}$ are as shown in part (a) of Fig. 3, and $M_{1}=16, M_{2}=M_{3}=12, M_{4}=9$. In the NE characterized in Theorem 4 , it turns out that $d^{\prime}, t_{1}, t_{2}, t_{3}$ and $t_{4}$ are independent ${ }^{16}$ of $q_{1}$, and their values for different $q_{2} \in(0,1)$ are as follows:

1) For $0<q_{2}<\frac{1}{4}, d^{\prime}=1, t_{1}=1, t_{2}=t_{3}=t_{4}=0$.

2) For $\frac{1}{4} \leq q_{2}<\frac{15}{16}, d^{\prime}=3, t_{1}=\frac{1}{11}\left(3+\frac{2}{q_{2}}\right), t_{2}=t_{3}=$ $\frac{1}{11}\left(4-\frac{1}{q_{2}}\right) t_{4}=0$.

3) For $\frac{15}{16} \leq q_{2}<1, d^{\prime}=4, t_{1}=\frac{1}{49}\left(9+\frac{13}{q_{2}}\right), t_{2}=t_{3}=$ $\frac{1}{49}\left(\frac{1}{q_{2}}+12\right) t_{4}=\frac{1}{49}\left(16-\frac{15}{q_{2}}\right)$.

Note that $t_{1} \geq t_{2} \geq t_{3} \geq t_{4}$ for each value of $q_{2}$, consistent with Theorem 4 . In fact, for all $q_{2}, t_{2}=t_{3}$, which is because $I_{2}$ and $I_{3}$ are of the same size. Fig. 7 plots $t_{1}, t_{2}$ and $t_{4}$ versus $q_{2}$. For small $q_{2}$, primaries offer bandwidth at the largest I.S. $I_{1}$ with probability 1 ; but as $q_{2}$ increases, the competition at

\footnotetext{
${ }^{16}$ This, in fact, holds in general because $d^{\prime}, t_{1}, \ldots, t_{d}$ are the solution of (23) and (24), which contain terms in the function $W(\alpha)=1-w_{1}(\alpha)$ and $w_{1}($.$) is independent of q_{1}$ by definition. However, the price distributions in the NE do depend on $q_{1}$
}

$I_{1}$ increases, inducing the primaries to shift probability mass from $I_{1}$ to the other I.S. So $t_{1}$ decreases in $q_{2}$. However, note that for all values of $q_{2}, t_{1} \geq t_{2} \geq t_{4}$ and $t_{4}$ is very small (less than 0.02).

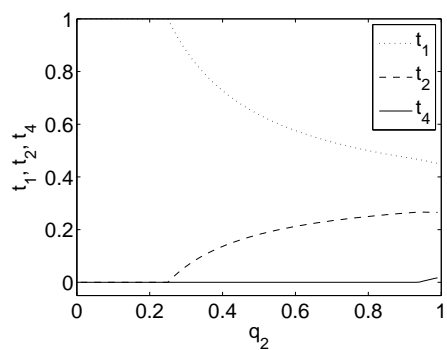

Fig. 7. The figure shows the NE probabilities $t_{1}, t_{2}$ and $t_{4}$ for the example in Section IV-C.

Finally, at the beginning of this section we showed that a system with multiple locations may have multiple NE. In fact, the example chosen was one where the conflict graph is linear, and is therefore mean valid by part 2 of Theorem 3 . Nevertheless, the NE in Theorem 4 turns out to be the unique one in a large class of strategy profiles- the class $\mathcal{S}$ in which every primary uses the same distribution (p.m.f.) to select the independent set at which to offer bandwidth.

Lemma 3: The NE characterized in Theorem 4 is unique in class $\mathcal{S}$.

Note that in a strategy profile in class $\mathcal{S}$, primaries may choose I.S. other than $I_{1}, \ldots, I_{d}$. The above lemma rules out the choice of any such I.S. under an NE.

\section{Threshold behavior}

We first define the efficiency, $\eta$, of a NE as $\eta=\frac{R_{\mathrm{NE}}}{R_{\mathrm{OPT}}}$, where $R_{\mathrm{NE}}$ is the expected sum of payoffs of the $n$ primaries at the $\mathrm{NE}$ and $R_{\mathrm{OPT}}$ is the maximum possible (optimal) expected sum of payoffs, attained when all primaries jointly select the independent sets and prices to maximize their aggregate revenue. Clearly, $\eta \leq 1$ quantifies the loss in aggregate revenue incurred owing to lack of cooperation among primaries. Also, since the above NE is unique (overall for the single location game and in class $\mathcal{S}$ for multiple locations), $\eta$ quantifies fundamental limits on the performance of $\mathrm{NE}$ in the respective categories.

Let $\lim _{n \rightarrow \infty} \sum_{i=1}^{n} \frac{q_{n}}{n}=q$ for some $q \in(0,1)$. Here, $q$ represents the "average" bandwidth availability probability of the primaries. For simplicity, we assume that each secondary from a given pool independently seeks bandwidth, and let $k_{n}$ be the expected number of secondaries at any given location ${ }^{17}$. Then, the NE structure exhibits interesting threshold behavior as $n \rightarrow \infty$; in particular, $\eta$ switches from 1 to 0 depending on the relations between $n q$ (availability) and $k_{n}$ (demand).

Lemma 4: Let ${ }^{18} \bar{q}_{n}=\frac{q_{1}+\ldots+q_{n}}{n}$ and let $\tilde{p}_{j}$ denote the common lower endpoint of the price distributions of the

\footnotetext{
${ }^{17} \mathrm{We}$ allow (but do not require) the number (rather statistics) of the secondaries to scale with increase in $n$.

${ }^{18}$ For simplicity, we state this lemma under the assumption that $M_{1}, \ldots, M_{d}$ are distinct. In the Appendix, we provide the lemma with this assumption relaxed.
} 
primaries who have unused bandwidth in the NE at nodes in I.S. $I_{j}$ (if they select I.S. $I_{j}$ ).

1) If there exists an $\epsilon>0$ such that for all large $n, q<$ $k_{n} /(n-1)-\epsilon$, then $\eta \rightarrow 1$ as $n \rightarrow \infty$. Also, for all large $n, d^{\prime}=1, t_{1}=1, t_{2}=t_{3}=\ldots t_{d}=0, \tilde{p}_{1} \rightarrow \nu$.

2) Let $l<d$. If there exists an $\epsilon>0$ such that for all large $n, l k_{n} /(n-1)+\epsilon<q<(l+1) k_{n} /(n-1)-\epsilon$, then for all large $n, d^{\prime} \geq l+1$, and $t_{j} \bar{q}_{n} \rightarrow k_{n} /(n-1)$ for all $j \leq l$.

3) If there exists an $\epsilon>0$ such that for all large $n, q>$ $k_{n} d /(n-1)+\epsilon$, then $\eta \rightarrow 0$ as $n \rightarrow \infty$. Also, for all large $n, d^{\prime}=d$ and $\tilde{p}_{j} \rightarrow c, j=1, \ldots, d$.

Intuitively, if availability is less than demand, then owing to limited competition, primaries with available bandwidth select only the maximum-sized I.S. among $I_{1}, \ldots, I_{d}$, and choose prices in a neighborhood of $\nu$. Thus, $\eta \rightarrow 1$, since no other strategy can enhance any primary's payoff. As availability increases, under NE, primaries diversify their choices among the I.S. $I_{1}, \ldots, I_{d}$ and are more likely to select low prices as well (the lower limits of the price distributions hover around $c$ once availability exceeds demand), thereby drastically reducing the efficiency of the NE.

\section{NUMERICAL STUdies}

In this section, we describe numerical computations that are directed towards assessing the impact of price competition among the primaries on the aggregate revenue of the primaries and the affordability of spectrum for the secondaries. We consider the specific case of a grid graph $\mathcal{H}_{m, m}$ (see Section IV-B2). By part 4 of Theorem 3, this is a mean valid graph and, in the notation of Definition 3, $d=4$ and the I.S. $I_{1}, I_{2}, I_{3}$ and $I_{4}$ are as shown in part (a) of Fig. 3. Throughout, we use the parameter values $\nu=1$ and $c=0$, and a constant number of secondaries $k$ at each node. Also, $q_{1}, \ldots, q_{n}$ are uniformly spaced in $\left[q_{L}, q_{H}\right]$ for some parameters $q_{L}$ and $q_{H}$. Let $q=\frac{q_{L}+q_{H}}{2}$ be the mean bandwidth availability probability of the primaries.

In $\mathcal{H}_{m, m}$, the NE is of the form in Theorem 4 and the plot on the left in Fig. 8 reveals, as expected, that price competition significantly reduces the aggregate revenue of the primaries under this NE relative to OPT, the optimal scheme in which the primaries collaborate to attain $R_{\mathrm{OPT}}$, the maximum aggregate revenue of the primaries (Note that under OPT, the I.S. $I_{1}, \ldots, I_{4}$ are selected in order of size and all the primaries always select the highest price $\nu$ ). Also, overall, the efficiency $(\eta)$ decreases as $q$ increases since the competition increases. The plot on the right in Fig. 8 shows that the trends are similar for a larger topology (larger $m$ ). The plot on the left in Fig. 9 shows that $\eta$ improves as $k$ increases. This is because, for small values of $k$, demand for bandwidth is scarce at each node. Under the NE, bandwidth is wasted at several nodes since $k+1$ or more primaries offer bandwidth at those nodes, resulting in a shortage of bandwidth at other nodes. On the other hand, since all primaries cooperate in OPT, it judiciously supplies bandwidth precisely where it is needed. So OPT outperforms the NE by a large margin for small values of $k$. For large values of $k$, the demand is high and so is the tolerable margin of error in assigning the primaries to I.S.; and hence the performance of the NE improves relative to OPT. The plot on the right in Fig. 9 shows that $\eta$ increases as $m$ increases, which is because the four I.S. $I_{1}, \ldots, I_{4}$ become closer to each other in size as $m$ increases and hence the loss in revenue resulting from choosing a smaller I.S. is lower.

Fig. 10 shows that under price competition, the expected price per unit of bandwidth is lower at the nodes in the larger I.S. This is because primaries prefer larger I.S. and hence the competition is more intense there, driving down the prices.
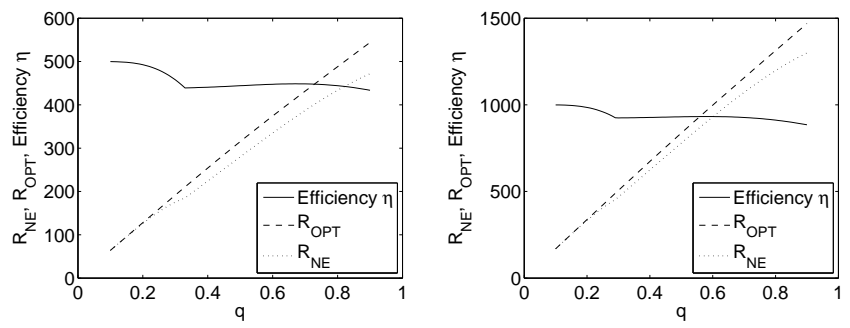

Fig. 8. Both figures plot the aggregate revenues of the primaries, $R_{N E}$ and $R_{O P T}$, under the NE and OPT respectively, and the efficiency of the NE, $\eta=\frac{R_{N E}}{R_{O P T}}$, versus $q$. In both figures, $n=10, k=5$ and $q_{H}-q_{L}=0.2$ are used. Also, $m=15$ (respectively, $m=25$ ) for the figure on the left (respectively, right). $\eta$ is scaled by a factor of 500 (respectively, 1000) in the figure on the left (respectively, right) in order to show it on the same figure as the other plots.
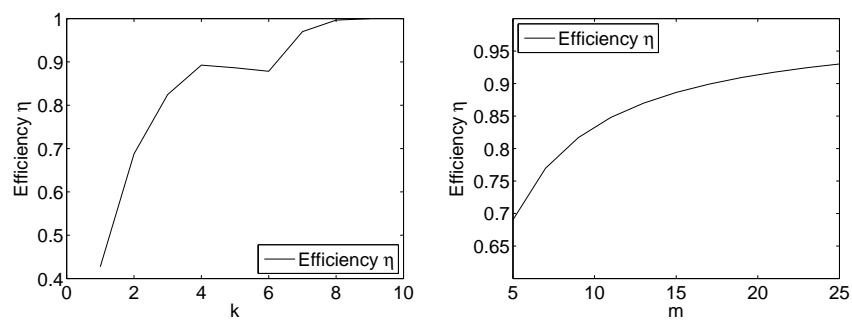

Fig. 9. The figure on the left (respectively, right) plots the efficiency $\eta$ of the NE versus $k$ (respectively, $m$ ). For both figures, $n=10, q_{L}=0$ and $q_{H}=1$ are used. Also, $m=15$ for the figure on the left and $k=5$ for the figure on the right.

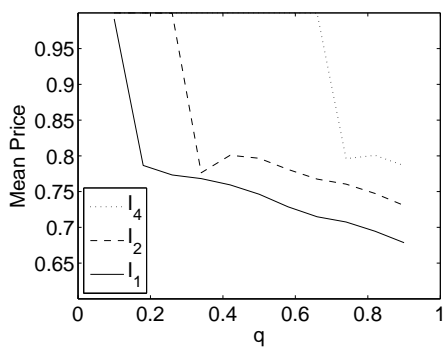

Fig. 10. The figure shows the mean price of bandwidth, given that it is offered, at a (fixed) node in each of $I_{1}, I_{2}$ and $I_{4}$ under the NE vs $q$. Note that since $\left|I_{3}\right|=\left|I_{2}\right|$, the mean price of bandwidth at nodes in $I_{3}$ is the same as that at nodes in $I_{2}$. The parameter values used are $m=15, n=8$ and $k=3$. Also, $q_{H}-q_{L}=0.2$. 


\section{REFERENCES}

[1] I. Akyildiz, W.-Y. Lee, M. Vuran, S. Mohanty "NeXt generation/dynamic spectrum access/cognitive radio wireless networks: a survey". In Comp. Networks, Vol. 50, 13, pp. 2127-59, 2006.

[2] R. Myerson, "Game Theory: Analysis of Conflict", Harvard University Press, 1997.

[3] J. E. Walsh, "Existence of Every Possible Distribution for any Sample Order Statistic", In Statistical Papers, Vol. 10, No. 3, Springer Berlin, Sept. 1969.

[4] W. Rudin, "Principles of Mathematical Analysis", Mc-Graw Hill, Third Edition, 1976.

[5] B.S. Everitt, The Cambridge Dictionary of Statistics, 3rd ed., Cambridge University Press, 2006.

[6] D. West, Introduction to Graph Theory, 2nd ed., Prentice Hall, 2000.

[7] A. Mas-Colell, M. Whinston, J. Green, "Microeconomic Theory", Oxford University Press, 1995.

[8] M. Osborne, C. Pitchik, "Price Competition in a Capacity-Constrained Duopoly", J. Econ. Theory, 38(2), 238-260, 1986.

[9] D.M. Kreps, J.A. Scheinkman, "Quantity Precommitment and Bertrand Competition Yield Cournot Outcomes", In Bell Journal of Economics, 14, pp. 326-337, Autumn 1983.

[10] M. Janssen, E. Rasmusen "Bertrand Competition Under Uncertainty", In J. Ind. Econ., 50(1): pp. 11-21, March 2002.

[11] S. Kimmel "Bertrand Competition Without Completely Certain Production", Economic Analysis Group Discussion Paper, Antitrust Division, U.S. Department of Justice, 2002.

[12] C. Courcoubetis and R. Weber, "Pricing Communication Networks", John Wiley \& Sons, Ltd. 2003.

[13] O. Ileri, D. Samardzija, T. Sizer, N. B. Mandayam, "Demand Responsive Pricing and Competitive Spectrum Allocation via a Spectrum Policy Server", In Proc. of IEEE DySpan, 2005.

[14] P. Maille, B. Tuffin "Analysis of Price Competition in a Slotted Resource Allocation Game", In Proc. of Infocom, 2008.

[15] P. Maille, B. Tuffin, "Price War with Partial Spectrum Sharing for Competitive Wireless Service Providers", In Proc. of IEEE Globecom, Dec. 2009.

[16] Y. Xing, R. Chandramouli, C. Cordeiro, "Price Dynamics in Competitive Agile Spectrum Access Markets", In IEEE JSAC, Vol. 25, No. 3, April 2007.

[17] D. Niyato, E. Hossain, "Competitive Pricing for Spectrum Sharing in Cognitive Radio Networks: Dynamic Game, Inefficiency of Nash Equilibrium, and Collusion", IEEE JSAC, Vol. 26, No. 1, 2008.

[18] D. Niyato, E. Hossain, Z. Han, "Dynamics of Multiple-Seller and Multiple-Buyer Spectrum Trading in Cognitive Radio Networks: A Game-Theoretic Modeling Approach", IEEE TMC, Vol. 8, No. 8, pp. 1009-1022, Aug. 2009.

[19] X. Zhou, H. Zheng, "TRUST: A General Framework for Truthful Double Spectrum Auctions", In Proc. of Infocom, 2009.

[20] S. Chawla and T. Roughgarden, "Bertrand Competition in Networks", In Symposium on Algorithmic Game Theory, 2008.

[21] E. Altman, A. Kumar, C. Singh, R. Sundaresan, "Spatial SINR Games Combining Base Station Placement and Mobile Association", In Proc. of Infocom, 2009.

[22] G.S. Kasbekar, S. Sarkar, "Spectrum Pricing Games with Bandwidth Uncertainty and Spatial Reuse in Cognitive Radio Networks", in Proc. of MobiHoc, 2010.

[23] L. Tassiulas, A. Ephremides, "Stability properties of constrained queuing systems and scheduling for maximum throughput in multihop radio networks”, IEEE TAC, Vol. 37, No. 12, pp. 1936-1949, Dec. 1992.

[24] T.S. Rappaport, "Wireless Communications: Principles and Practices", 2nd ed, Prentice Hall, 2002.

[25] G.S. Kasbekar, S. Sarkar, "Spectrum Pricing Games with Arbitrary Bandwidth Availability Probabilities", In Proc. of ISIT, St. Petersburg, Russia, July 31-August 5, 2011.

[26] G.S. Kasbekar, S. Sarkar, "Spectrum Pricing Games with Spatial Reuse in Cognitive Radio Networks", submitted. Technical report available at: http://www.seas.upenn.edu/ swati/publication.htm

[27] W. Hoeffding, "Probability inequalities for sums of bounded random variables", Journal of the American Statistical Association 58 (301): 1330, March 1963.

\section{APPENDIX}

\section{A. Proofs of results in Section III-A}

We first prove a series of lemmas and then deduce Properties 1 and 2 and Theorem 1 from them.
Lemma 5: For $i=1, \ldots, n, \psi_{i}($.$) is continuous, except$ possibly at $v$. Also, at most one primary has a jump at $v$.

Proof: Suppose $\psi_{i}($.$) has a jump at a point x_{0}, c<x_{0}<$ $v$. Then for some $\epsilon>0$, no primary $j \neq i$ chooses a price in $\left[x_{0}, x_{0}+\epsilon\right]$ because it can get a strictly higher payoff by choosing a price just below $x_{0}$ instead. This in turn implies that primary $i$ gets a strictly higher payoff at the price $x_{0}+\epsilon$ than at $x_{0}$. So $x_{0}$ is not a best response for primary $i$, which contradicts the assumption that $\psi_{i}($.$) has a jump at x_{0}$. Thus, $\psi_{i}($.$) is continuous at all x<v$.

Now, suppose primary $i$ has a jump at $v$. Then a primary $j \neq i$ gets a higher payoff at a price just below $v$ than at $v$. So $v$ is not a best response for primary $j$ and it plays it with 0 probability. Thus, at most one primary has a jump at $v$.

Lemma 6: For every $\epsilon>0$, there exist primaries $m$ and $j$, $m \neq j$, such that $\psi_{m}(v-\epsilon)<1$ and $\psi_{j}(v-\epsilon)<1$.

That is, at least two primaries play prices just below $v$ with positive probability.

Proof: Suppose not. Fix $i$ and let:

$$
y=\inf \left\{x: \psi_{l}(x)=1 \forall l \neq i\right\} .
$$

By definition of $y, \psi_{l}(x)=1 \forall l \neq i$ and $x>y$. Also, since $\psi_{l}($.$) is a distribution function, it is right continuous [5]. So$

$$
\psi_{l}(y)=1 \forall l \neq i \text {. }
$$

Suppose $y<v$. By (26):

$$
P\left\{p_{l} \in(y, v]\right\}=0, \forall l \neq i .
$$

So every price $p_{i} \in(y, v)$ is dominated by $p_{i}=v$. Hence:

$$
P\left\{p_{i} \in(y, v)\right\}=0
$$

By (27) and (28):

$$
P\left\{p_{j} \in(y, v)\right\}=0, j=1, \ldots, n .
$$

By (25), $\forall \epsilon>0, \psi_{l}(y-\epsilon)<1$ for at least one primary $l \neq i$; otherwise the infimum in the RHS of (25) would be less than $y$. So this primary $l$ plays prices just below $y$ with positive probability. Now, if primary $l$ sets a price $p_{l}<v$, it gets a payoff equal to the revenue, $\left(p_{l}-c\right)$, if bandwidth is sold, times the probability that bandwidth is sold. Also, by Lemma 5, $\psi_{j}(),. j=1, \ldots, n$ are continuous at all prices below $v$. So by (29), a price $p_{l}$ just below $v$ yields a higher payoff than a price just below $y$. This is because, $p_{l}-c$ is lower by approximately $v-y$ for $p_{l}$ just below $y$ than for $p_{l}$ just below $v$, but by (29) and continuity of $\psi_{j}(),. j=1, \ldots, n$, the probability that bandwidth is sold for a price $p_{l}$ just below $y$ can be made arbitrarily close to the probability that bandwidth is sold for a price $p_{l}$ just below $v$. This contradicts the assumption that primary $l$ plays prices just below $y$ with positive probability.

Thus, $y$ in (25) equals $v$ and hence at least one primary $j \neq i$ plays prices just below $v$ with positive probability. The above arguments with $j$ in place of $i$ imply that at least one primary other than $j$ plays prices just below $v$ with positive probability. Thus, at least two primaries in $\{1, \ldots, n\}$ play prices just below $v$ with positive probability.

Let $u_{i, \max }$ and $L_{i}$ be as defined in Section III-A.

Lemma 7: For $i=1, \ldots, n, L_{i}$ is a best response for primary $i$. 
Proof: By (3), either primary $i$ has a jump at $L_{i}$ or plays prices arbitrarily close to $L_{i}$ and above it with positive probability.

Case (i): If primary $i$ has a jump at $L_{i}$, then $L_{i}$ is a best response for $i$ because in a NE, no primary plays a price other than a best response with positive probability.

Case (ii): If primary $i$ does not have a jump at $L_{i}$, then by (3), $\psi_{i}\left(L_{i}\right)=0$. Since every primary selects a price in $[c, v]$, $\psi_{i}(v)=1$. So $L_{i}<v$. So by Lemma 5, no primary among $\{1, \ldots, n\} \backslash i$ has a jump at $L_{i}$. Hence, primary $i$ 's payoff at a price above $L_{i}$ and close enough to it is arbitrarily close to its payoff at $L_{i}$. But since primary $i$ does not have a jump at $L_{i}$, by (3), it plays prices just above $L_{i}$ with positive probability and they are best responses for him. So $L_{i}$ is also a best response for primary $i$.

Lemma 8: For some $c<\tilde{p}<v, L_{1}=\ldots L_{n}=\tilde{p}$. Also, $u_{i, \max }=(\tilde{p}-c) r, i=1, \ldots, n$.

That is, the lower endpoint of the support set of the price distribution of every primary is the same.

Proof: Let $L_{\min }=\min \left\{L_{m}: m=1, \ldots, n\right\}$, and $S_{\min }=\left\{m: L_{m}=L_{\min }\right\}$ be the set of primaries with the lowest endpoint. Let

$$
k_{\min }=\min _{k}\left\{k: \gamma_{k}>0\right\} .
$$

Thus, $k_{\min }$ is the minimum number of secondaries at a location. Note that $k_{\min }$ will be 0 if $\gamma_{0}>0$, and $k_{\min }>0$ otherwise. First, we show by contradiction that:

$$
\left|S_{\min }\right| \geq k_{\min }+1 \text {. }
$$

Clearly, the above holds if $k_{\min }=0$. We therefore show that it holds even otherwise. Suppose $\left|S_{\min }\right| \leq k_{\min }$. If $L_{\min }=v$, then all primaries play the price $v$ w.p. 1 , which does not constitute a NE by Lemma 5. So $L_{\min }<v$ and again by Lemma 5, no primary has a jump at $L_{m i n}$. Also, by Lemma 7, $L_{\min }$ is a best response for the primaries in $S_{\min }$. Let $\hat{L}=$ $\min \left\{L_{m}: m \notin S_{m i n}\right\}$ be the second lowest endpoint. Now, a primary $m \in S_{\min }$ who has unused bandwidth can get a higher payoff at a price just below $\hat{L}$ than at $L_{\min }$ because in both cases, since $\left|S_{\min }\right| \leq k_{\min }$, primary $m$ 's bandwidth is sold w.p. 1; however, it gets a higher revenue at a price just below $L$ than at $L_{\min }$. This contradicts the fact that $L_{\min }$ is a best response for primary $m$. Thus, (30) must hold.

Now, suppose $L_{i}<L_{j}$ for some $i, j$. By Lemma $7, L_{j}$ is a best response for primary $j$. Now, the expected payoff that primary $j$ gets for $p_{j}=L_{j}$ is strictly less than the expected payoff that primary $i$ would get if it set $p_{i}$ to be just below $L_{j}$. This is because, if primaries $i$ or $j$ set a price of approximately $L_{j}$, then they see the same price distribution functions of the primaries other than $i$ and $j$. But primary $j$ may be undercut by primary $i$, since $L_{i}<L_{j}$, whereas primary $i$ may not be undercut by primary $j$. Also, by (30), primary $j$ 's expected payoff is strictly lowered due to this undercutting by primary $i$. (Note that if $k_{\min }>0$, undercutting by primary $i$ would not lower primary $j$ 's probability of winning, and thereby the expected payoff, if a total of $\leq k_{\min }-1$ primaries played prices below $L_{j}$ with positive probability. This possibility is ruled out by (30). If $k_{\min }=0, \gamma_{0}>0$. If in addition $\gamma_{1}=0$, and $S_{\min }=$ 1 , it is possible that only 1 primary (i.e., $i$ ) plays prices below
$L_{j}$ with positive probability. In this case, note that whenever at least 1 secondary is available, at least 2 secondaries are available (as $\gamma_{1}=0$ ), and hence undercutting by primary $i$ does not lower primary $j$ 's probability of winning, and thereby the expected payoff. This possibility is ruled out by assumption (ii) on $\left\{\gamma_{k}\right\}$ in Section II-A since $\gamma_{1}>0$ if $\gamma_{0}>0$.) Hence, $u_{i, \max }>u_{j, \max }$.

Now, by Lemma 7, $L_{i}$ is a best response for primary $i$. If primary $j$ were to play price $L_{i}$, then it would get a payoff of $u_{i, \max }$. This is because, when primary $i$ plays price $L_{i}$, it gets payoff $u_{i, \max }$. Since $L_{j}>L_{i}$, primary $i$ is, w.p. 1 , not undercut by primary $j$. If primary $j$ were to set the price $L_{i}$, then w.p. 1 , it would not be undercut by primary $i$. Also, the price distributions of the primaries other than $i$ and $j$ are exactly the same from the viewpoints of primaries $i$ and $j$. Thus, primary $j$ can strictly increase its payoff from $u_{j, \max }$ to $u_{i, \max }$ by playing price $L_{i}$, which contradicts the fact that $L_{j}$ is a best response for him.

Thus, $L_{i}<L_{j}$ is not possible. By symmetry, $L_{i}>L_{j}$ is not possible. So $L_{i}=L_{j}$. Let $L_{1}=\ldots=L_{n}=\tilde{p}$.

If $\tilde{p}=v$, then every primary plays the price $v$ w.p. 1, which does not constitute a NE. So $\tilde{p}<v$. So by Lemma 5 , no primary has a jump at $\tilde{p}$. Thus, since the lower endpoint of the support set of every primary is $\tilde{p}$, by (3), a price of $\tilde{p}$ is a best response for every primary $i$. Since no primary sets a price lower than $\tilde{p}$, a price of $\tilde{p}$ fetches a payoff of $\tilde{p}-c$ if $K \geq 1$ and a payoff of 0 if $K=0$. So $u_{i, \max }=(\tilde{p}-c) P(K \geq$ 1) $=(\tilde{p}-c) r, i=1, \ldots, n$.

Let $w_{i}$ be as defined in Section III-A. Using (1), it can be easily shown that:

$$
w_{1} \leq w_{2} \leq \ldots \leq w_{n}
$$

Lemma 9: $\tilde{p}=c+\frac{\left(1-w_{1}\right)(v-c)}{r}$.

Proof: If primary 1 sets the price $p_{1}=v$, then it gets an expected payoff of at least $(v-c)\left(1-w_{1}\right)$ because its bandwidth is sold at least in the event that $k-1$ or fewer primaries out of $2, \ldots, n$ have unused bandwidth. So $u_{1, \max } \geq$ $(v-c)\left(1-w_{1}\right)$. Since $u_{1, \max }=(\tilde{p}-c) r$ by Lemma 8 , we get:

$$
\tilde{p} \geq c+\frac{\left(1-w_{1}\right)(v-c)}{r} .
$$

Now, by Lemma 6, at least two primaries, say $m$ and $j$, play prices just below $v$ with positive probability. By Lemma 5, at most one of them has a jump at $v$. So assume, WLOG, that no primary other than $j$ has a jump at $v$. Then a price of $p_{j}=v$ is a best response for primary $j$ and fetches a payoff of $u_{j, \max }=(v-c)\left(1-w_{j}\right) \leq(v-c)\left(1-w_{1}\right)$, where the inequality follows from (31). Since $u_{j, \max }=(\tilde{p}-c) r$ by Lemma 8, we get:

$$
\tilde{p} \leq c+\frac{\left(1-w_{1}\right)(v-c)}{r} .
$$

The result follows from (32) and (33).

Lemma 10: Let $\tilde{p} \leq a<b \leq v$. Then at least two primaries play prices in $(a, b)$ with positive probability.

Proof: If $b=v$, then the claim is true by Lemma 6. If $a=\tilde{p}$, then the claim is true by Lemma 5 and Lemma 8, since $\tilde{p}<v$ is the lower endpoint of the support set of all 
primaries and no primary has a jump at $\tilde{p}$; hence all primaries play prices just above $\tilde{p}$ with positive probability.

Now, fix any $a, b$ such that $\tilde{p}<a<b<v$. Let:

$$
\underline{a}=\inf \left\{x \leq a: \psi_{j}(x)=\psi_{j}(a) \forall j=1, \ldots, n\right\}
$$

By Lemma 8, $\underline{a}>\tilde{p}$. Also, by definition of $\underline{a}, P\left\{p_{j} \in\right.$ $[\underline{a}, a]\}=0 \forall j=1, \ldots, n$.

By definition of $\underline{a}$, at least one primary, say primary $i$, plays prices just below $\underline{a}$ with positive probability. (If not, then the infimum in (34) would be less than $a$.) This implies that at least one primary $j \neq i$ plays prices in $(\underline{a}, b)$ with positive probability. (If not, then $p_{i}=b$ would yield a strictly higher payoff to primary $i$ than prices just below $\underline{a}$.) Now, if primary $j$ is the only primary among primaries $\{1, \ldots, n\}$ who play prices in $(\underline{a}, b)$ with positive probability, then $p_{j}=b$ yields a strictly higher payoff than $p_{j} \in(\underline{a}, b)$, which is a contradiction. So at least two primaries play prices in $(\underline{a}, b)$ with positive probability. But $P\left\{p_{l} \in[\underline{a}, a]\right\}=0 \forall l=1, \ldots, n$ by definition of $\underline{a}$. Hence, at least two primaries play prices in $(a, b)$ with positive probability.

Let $F_{-i}(x)$ be as defined in Section III-B.

Lemma 11: For a fixed $x \in(\tilde{p}, v]$, and primaries $i$ and $j$, (i) $F_{-i}(x)=F_{-j}(x)$ iff $\phi_{i}(x)=\phi_{j}(x)$, (ii) $F_{-i}(x)<F_{-j}(x)$ iff $\phi_{i}(x)>\phi_{j}(x)$.

Proof: Let $p_{(l)}^{\prime}$ be the l'th smallest out of the pseudoprices of the primaries other than $i$ and $j$. Let $F_{-i, k}(x)$ be the probability that $p_{-i}^{\prime} \leq x$ given that $K=k$. Clearly, $F_{-i, 0}(x)=1$ since $x>\tilde{p} \geq 0$, and $F_{-i, k}(x)=0$ if $k>n-1$. We evaluate $F_{-i, k}(x)$ for $1 \leq k \leq n-1$. Conditioning on the event $\left\{p_{j}^{\prime} \leq x\right\}$ and using the fact that $\left\{p_{l}^{\prime}: l \neq i\right\}$ are independent, we get:

$$
\begin{aligned}
F_{-i, k}(x) & \\
= & P\left\{k^{\prime} \text { th smallest of }\left\{p_{l}^{\prime}: l \neq i\right\} \leq x\right\} \\
= & P\left\{p_{j}^{\prime} \leq x\right\} P\left\{p_{(k-1)}^{\prime} \leq x\right\}+P\left\{p_{j}^{\prime}>x\right\} P\left\{p_{(k)}^{\prime} \leq x\right\} \\
= & \phi_{j}(x) P\left\{p_{(k-1)}^{\prime} \leq x\right\}+\left(1-\phi_{j}(x)\right) P\left\{p_{(k)}^{\prime} \leq x\right\} \\
= & \phi_{j}(x)\left[P\left\{p_{(k-1)}^{\prime} \leq x\right\}-P\left\{p_{(k)}^{\prime} \leq x\right\}\right] \\
& +P\left\{p_{(k)}^{\prime} \leq x\right\}
\end{aligned}
$$

Similarly,

$F_{-j, k}(x)=\phi_{i}(x)\left[P\left\{p_{(k-1)}^{\prime} \leq x\right\}-P\left\{p_{(k)}^{\prime} \leq x\right\}\right]+P\left\{p_{(k)}^{\prime} \leq x\right.$

By (35) and (36):

$$
\begin{aligned}
& F_{-i, k}(x)-F_{-j, k}(x) \\
& \quad=\left(\phi_{j}(x)-\phi_{i}(x)\right)\left[P\left\{p_{(k-1)}^{\prime} \leq x\right\}-P\left\{p_{(k)}^{\prime} \leq x\right\}\right] \\
& \quad=\left(\phi_{j}(x)-\phi_{i}(x)\right) \alpha_{k}
\end{aligned}
$$

where $\alpha_{k}=P\left\{p_{(k-1)}^{\prime} \leq x\right\}-P\left\{p_{(k)}^{\prime} \leq x\right\}$. Thus,

$$
F_{-i}(x)-F_{-j}(x)=\left(\phi_{j}(x)-\phi_{i}(x)\right) \sum_{k=1}^{n-1} \alpha_{k} \gamma_{k} .
$$

We will next show that $\alpha_{k}>0$ for $1 \leq k \leq n-1$. Both parts of the result will then follow from the above.

Note that $\alpha_{k}$ equals the probability that exactly $(k-1)$ out of the pseudo-prices of the primaries other than $i$ and $j$ are $\leq x$. Since $x>\tilde{p}$, all primaries play prices in $(\tilde{p}, x)$ with positive probability by Lemma 8 . So:

$$
\phi_{l}(x)=P\left\{p_{l}^{\prime} \leq x\right\}>0, l=1, \ldots, n .
$$

Also,

$$
\phi_{l}(x) \leq \phi_{l}(v)=q_{l}<1, l=1, \ldots, n .
$$

By (38) and (39):

$$
0<\phi_{l}(x)<1, l=1, \ldots, n .
$$

Also, since $1 \leq k \leq n-1$, we have:

$$
0 \leq k-1 \leq n-2
$$

Since $\alpha_{k}$ equals the probability of exactly $k-1$ successes out of $n-2$ independent Bernoulli events that have success probabilities $\left\{\phi_{l}(x): l=1, \ldots, n, l \neq i, j\right\}, \alpha_{k}>0$ by (40) and (41). This completes the proof.

Lemma 12: (i) $\phi_{2}(),. \ldots, \phi_{n}($.$) are continuous at v$. (ii) $\phi_{1}($.$) is continuous at v$ if $q_{1}=q_{2}$ and has a jump of size at most $q_{1}-q_{2}$ at $v$ if $q_{1}>q_{2}$. Also,

$$
\phi_{1}(v-) \geq q_{2} \text {. }
$$

Proof: If no primary $i>1$ has a jump at $v$, then primary 1 gets a payoff of $(v-c)\left(1-w_{1}\right)$, which equals $(\tilde{p}-c) r$ by Lemma 9, for a price $p_{1}$ just below $v$ in the limit as $p_{1} \rightarrow v-$. So if a primary $i \geq 2$ has a jump at $v$, primary 1 can get a payoff strictly greater than $(\tilde{p}-c) r$ by playing a price close enough to $v$. This contradicts the fact that $u_{1, \max }=(\tilde{p}-c) r$ (see Lemma 8). Thus, no primary $i \geq 2$ has a jump at $v$ and $\phi_{2}(),. \ldots, \phi_{n}($.$) are continuous.$

First, suppose $q_{1}=q_{2}$. If primary 1 has a jump at $v$, then similar to the preceding paragraph, primary 2 can get a payoff strictly greater than $(\tilde{p}-c) r$ by playing a price just below $v$, which contradicts the fact that $u_{2, \max }=(\tilde{p}-c) r$. So $\psi_{1}($.$) is$ continuous.

Now suppose $q_{1}>q_{2}$. First, suppose primary 1 has a jump of size exactly $q_{1}-q_{2}$ at $v$. Then if primary 2 sets a price just below $v$, then the probability of being undercut by primary $j \in\{3, \ldots, n\}$ is approximately $q_{j}$. Also, since primary 1 has a jump of size $q_{1}-q_{2}$ at $v$, the probability of being undercut by primary 1 is approximately $q_{1}-\left(q_{1}-q_{2}\right)=q_{2}$. So at a price just below $v$, primary 2 sees the same set of probabilities of being undercut by primaries other than itself as primary 1 would see if it set a price just below $v$. Hence, by the first paragraph of this proof, primary 2 gets a payoff of approximately $(\tilde{p}-c) r$ at a price just below $v$.

Hence, if primary 1 has a jump of size, not equal to, but greater than $q_{1}-q_{2}$ at $v$, primary 2 gets a payoff of strictly greater than $(\tilde{p}-c) r$ at a price just below $v$. This contradicts the fact that $u_{2, \max }=(\tilde{p}-c) r$.

Thus, primary 1 has a jump of at most size $q_{1}-q_{2}$ at $v$. So $\phi_{1}(v)-\phi_{1}(v-) \leq q_{1}-q_{2}$. This, along with $\phi_{1}(v)=q_{1}$, gives (42).

Lemma 13: If $\tilde{p} \leq x<y<v$ and $\psi_{i}(x)=\psi_{i}(y)$ for some primary $i$, then $\psi_{i}(v-)=\psi_{i}(x)$.

Thus, if $x \geq \tilde{p}$ is the left endpoint of an interval of constancy of $\psi_{i}($.$) for some i$, then to the right of $x$, the interval of constancy extends at least until $v$ (there may be a jump at $v$ ). 
Proof: Suppose not, i.e.:

$$
\psi_{i}(v-)>\psi_{i}(x) .
$$

Let:

$$
\bar{y}=\sup \left\{z \geq x: \psi_{i}(z)=\psi_{i}(x)\right\}
$$

By (43), (44) and the fact that $\psi_{i}($.$) is continuous below v$ (by Lemma 5), we get $\bar{y}<v$. So again by Lemma 5 , no primary among $\{1, \ldots, n\} \backslash i$ has a jump at $\bar{y}$. Also, primary $i$ uses prices just above $\bar{y}$ with positive probability (if not, the supremum in the RHS of (44) would be $>\bar{y}$ ). So $\bar{y}$ is a best response for primary $i$ and hence:

$$
E\left\{u_{i}\left(\bar{y}, \psi_{-i}\right)\right\}=(\bar{y}-c)\left(1-F_{-i}(\bar{y})\right)=u_{i, \max }=(\tilde{p}-c) r .
$$

where the last equality follows from Lemma 8 .

Now, by Lemma 10 , there exists a primary $j \neq i$ who plays prices just below $\bar{y}$ with positive probability. Since no primary among $\{1, \ldots, n\} \backslash j$ has a jump at $\bar{y}, \bar{y}$ is a best response for primary $j$. Hence:

$$
E\left\{u_{j}\left(\bar{y}, \psi_{-j}\right)\right\}=(\bar{y}-c)\left(1-F_{j}(\bar{y})\right)=u_{j, \max }=(\tilde{p}-c) r .
$$

By (45) and (46), $F_{-i}(\bar{y})=F_{-j}(\bar{y})$. So by Lemma 11 :

$$
\phi_{i}(\bar{y})=\phi_{j}(\bar{y}) .
$$

But since primary $j$ plays prices just below $\bar{y}$ with positive probability, there exists $\epsilon>0$ such that $x<\bar{y}-\epsilon$ and $\bar{y}-\epsilon$ is a best response for primary $j$. So

$$
\phi_{j}(\bar{y}-\epsilon)<\phi_{j}(\bar{y}) .
$$

But by (44) and the continuity of $\phi_{i}($.$) at \bar{y}$ :

$$
\phi_{i}(\bar{y})=\phi_{i}(\bar{y}-\epsilon) .
$$

By (47), (48) and (49), $\phi_{i}(\bar{y}-\epsilon)>\phi_{j}(\bar{y}-\epsilon)$. So by Lemma 11:

$$
F_{-j}(\bar{y}-\epsilon)>F_{-i}(\bar{y}-\epsilon)
$$

This implies:

$$
\begin{aligned}
(\tilde{p}-c) r & =E\left\{u_{j}\left(\bar{y}-\epsilon, \psi_{-j}\right)\right\} \\
& =(\bar{y}-\epsilon-c)\left(1-F_{-j}(\bar{y}-\epsilon)\right) \\
& <(\bar{y}-\epsilon-c)\left(1-F_{-i}(\bar{y}-\epsilon)\right) \\
& =E\left\{u_{i}\left(\bar{y}-\epsilon, \psi_{-i}\right)\right\}
\end{aligned}
$$

which contradicts the fact that every primary gets a payoff of $(\tilde{p}-c) r$ at a best response in the NE.

Lemma 14: Part 2 of Theorem 1 holds.

Proof: We prove the result by induction. Let:

$$
R_{n}=\inf \left\{x \geq \tilde{p}: \exists y>x \text { and } i \text { s.t. } \phi_{i}(y)=\phi_{i}(x)\right\}
$$

Note that $R_{n}$ is the smallest value $\geq \tilde{p}$ that is the left endpoint of an interval of constancy for some $\phi_{i}($.$) . For this i, \phi_{i}\left(R_{n}\right)=$ $\phi_{i}(y)$ for some $y>R_{n}{ }^{19}$. We must have $R_{n}>\tilde{p}$. This is because, if $R_{n}=\tilde{p}$, then $\phi_{i}(y)=\phi_{i}(\tilde{p})$. But $\phi_{i}(\tilde{p})=0$, since $\tilde{p}$ is the lower endpoint of the support set of $\phi_{i}($.$) by Lemma 8$. So $\phi_{i}(y)=0$, which implies that the lower endpoint of the

\footnotetext{
${ }^{19}$ Note that $\phi_{i}($.$) is a distribution function and hence is right continuous [5].$ So $\phi_{i}\left(R_{n}+\right)=\phi_{i}\left(R_{n}\right)$.
}

support set of $\phi_{i}($.$) is \geq y>\tilde{p}$. This contradicts Lemma 8 . Thus, $R_{n}>\tilde{p}$.

Now, by definition of $R_{n}$, all primaries play every subinterval in $\left[\tilde{p}, R_{n}\right)$ with positive probability and hence every price $x \in\left[\tilde{p}, R_{n}\right)$ is a best response for every primary. So similar to the derivation of (8), for $j \in\{1, \ldots, n\}$ and $x \in$ $\left[\tilde{p}, R_{n}\right), E\left\{u_{j}\left(x, \psi_{-j}\right)\right\}=(x-c)\left(1-F_{-j}(x)\right)=(\tilde{p}-c) r$. Hence, $F_{-1}(x)=\ldots=F_{-n}(x)$ and by Lemma 11 ,

$$
\phi_{1}(x)=\ldots=\phi_{n}(x)=\phi(x) \text { (say), } \tilde{p} \leq x<R_{n} .
$$

which proves (5) for $j=n$.

Case (i): Suppose $R_{n}=v$. Then $\phi_{l}\left(R_{n}\right)=q_{l}, l=1, \ldots, n$ (since $\psi_{l}(v)=1$ ), which proves (6).

Case (ii): Now suppose $R_{n}<v$. Then $\phi_{j}(),. j=1, \ldots, n$ are continuous at $R_{n}$ by Lemma 5 . So by (51):

$$
\phi_{1}\left(R_{n}\right)=\phi_{2}\left(R_{n}\right)=\ldots=\phi_{n}\left(R_{n}\right) .
$$

Since $R_{n}$ is the left endpoint of an interval of constancy of $\phi_{i}($.$) , by Lemma 13$ :

$$
\phi_{i}\left(R_{n}\right)=\phi_{i}(v-)=\phi_{n}\left(R_{n}\right) \leq q_{n}
$$

where the second equality follows from (52).

Now, suppose $i=1$. Then by (42) and (53):

$$
\phi_{i}\left(R_{n}\right) \geq q_{2} .
$$

By (53), (54) and (1), $q_{2}=q_{3}=\ldots=q_{n}=\phi_{i}\left(R_{n}\right)$. Also, by (52), $\phi_{j}\left(R_{n}\right)=q_{j}, j=2, \ldots, n$. So $\psi_{j}\left(R_{n}\right)=1$, $j=2, \ldots, n$. This implies, since $R_{n}<v$ by assumption, that at most one primary (primary 1) plays prices in the interval $\left(R_{n}, v\right)$ with positive probability, which contradicts Lemma 6. Thus, $i \neq 1$.

So by Lemma 12, $\phi_{i}($.$) is continuous at v$ and $\phi_{i}(v-)=$ $\phi_{i}(v)=q_{i}$. So by (53):

$$
\phi_{i}\left(R_{n}\right)=q_{i} .
$$

By (52) and (55), $\phi_{n}\left(R_{n}\right)=q_{i}$. If $q_{i}>q_{n}$, then $\phi_{n}\left(R_{n}\right)>q_{n}$, which is a contradiction because $\phi_{n}\left(R_{n}\right)=q_{n} \psi_{n}\left(R_{n}\right) \leq q_{n}$. So $q_{i} \leq q_{n}$. Also, since $q_{i} \geq q_{n}$ by (1), $q_{i}=q_{n}$. So:

$$
\phi_{n}\left(R_{n}\right)=q_{n} .
$$

which proves (6) for $j=n$.

Now, as induction hypothesis, suppose there exist thresholds:

$$
\tilde{p}<R_{n} \leq R_{n-1} \leq \ldots \leq R_{i+1} \leq v
$$

such that for each $j \in\{i+1, \ldots, n\}, \phi_{j}\left(R_{j}\right)=q_{j}$,

$$
\phi_{1}(x)=\ldots=\phi_{j}(x)=\phi(x), \tilde{p} \leq x<R_{j},
$$

and each of primaries $1, \ldots, j$ plays every sub-interval in $\left[\tilde{p}, R_{j}\right)$ with positive probability.

First, suppose $R_{i+1}<v$. Let:

$$
\begin{aligned}
R_{i}= & \inf \left\{x \geq R_{i+1}: \exists y>x \text { and } j \in\{1, \ldots, i\}\right. \\
& \text { s.t. } \left.\phi_{j}(y)=\phi_{j}(x)\right\} .
\end{aligned}
$$

If $R_{i}=R_{i+1}$, then clearly by (57):

$$
\phi_{1}(x)=\ldots=\phi_{i}(x)=\phi(x), \tilde{p} \leq x<R_{i}
$$


which proves (5) for $j=i$. Also, similar to (56), it can be shown that $\phi_{i}\left(R_{i}\right)=q_{i}$, which proves (6) for $j=i$ and completes the inductive step. Now suppose $R_{i}>R_{i+1}$. Then similar to the proof of (51), it can be shown that:

$$
\phi_{1}(x)=\ldots=\phi_{i}(x)=\phi(x), R_{i+1} \leq x<R_{i} .
$$

By (57) and (59):

$$
\phi_{1}(x)=\ldots=\phi_{i}(x)=\phi(x), \tilde{p} \leq x<R_{i} .
$$

which proves (5) for $j=i$. Also, similar to the proof of (56), it can be shown that $\phi_{i}\left(R_{i}\right)=q_{i}$, which proves (6) for $j=i$. This completes the induction.

If $R_{i+1}=v$, then the induction is completed by simply setting $R_{1}=\ldots=R_{i}=v$.

It remains to show that $R_{1}=R_{2}=v$. If $R_{1}<v$, then no primary plays a price in $\left(R_{1}, v\right)$, which contradicts Lemma 6 . So $R_{1}=v$. If $R_{2}<v$, then only primary 1 plays prices in $\left(R_{2}, v\right)$ with positive probability, which again contradicts Lemma 6. So $R_{2}=v$.

Lemma 15: If $q_{1}>q_{2}$, then $\phi_{1}($.$) has a jump of size q_{1}-q_{2}$ at $v$. So:

Proof: By Lemma 14, $\phi_{1}(x)=\phi_{2}(x)$ for all $x<R_{2}=v$.

$$
\begin{aligned}
\phi_{1}(v-) & =\phi_{2}(v-) \\
& =\phi_{2}(v) \quad\left(\text { since } \phi_{2}(.) \text { is continuous by Lemma } 12\right) \\
& =q_{2}
\end{aligned}
$$

Also, $\phi_{1}(v)=q_{1} \psi_{1}(v)=q_{1}$. So $\phi_{1}(v)-\phi_{1}(v-)=q_{1}-q_{2}$.

Finally, (i) Property 1 follows from Lemmas 5, 12 and 15; (ii) Property 2 follows from Lemmas 8 and 9; (iii) Theorem 1 follows from Properties 1 and 2 and Lemma 14.

\section{B. Proofs of results in Section III-B}

We verify that with $R_{i}$ as in (13), $R_{i} \geq R_{i+1}$ as required by (4) in Theorem 1. Recall from Section III-B1 that $f_{i}\left(q_{i}\right)$ is the probability of $K$ or more successes out of $n-1$ independent Bernoulli events, $i-1$ with success probability $q_{i}$ and $n-i$ with $q_{i+1}, \ldots, q_{n}$. Also, $f_{i+1}\left(q_{i+1}\right)$ is the probability of $K$ or more successes out of $n-1$ Bernoulli events, $i-1$ with success probability $q_{i+1}$ and $n-i$ with $q_{i+1}, \ldots, q_{n}$. Since $q_{i} \geq q_{i+1}$ by (1), it is easy to check that $f_{i}\left(q_{i}\right) \geq f_{i+1}\left(q_{i+1}\right)$. So by (13), $R_{i} \geq R_{i+1}$, which is consistent with (4).

Proof of Lemma 1: First, let $f_{i}($.$) be as defined in$ Section III-B1. To compute $f_{i}(y)$, for $i \in\{2, \ldots, n\}$, let $f_{i, k}(y)$ be the conditional probability given $K=k$, of $K$ or more successes out of $n-1$ independent Bernoulli events, $(i-1)$ of which have the same success probability $y$ and the remaining $(n-i)$ have success probabilities $q_{i+1}, \ldots, q_{n}$. Clearly,

$$
f_{i}(y)=\sum_{k} f_{i, k}(y) \gamma_{k}
$$

Again, $f_{i, 0}(y)=1$ and $f_{i, k}(y)=0$ if $k>n-1$.

Consider $1 \leq k \leq n-1$. For $l \in\{0, \ldots, n-i\}$, let $v_{l}^{i}\left(q_{i+1}, \ldots, q_{n}\right)$ be the probability of exactly $l$ successes out of $n-i$ independent Bernoulli trials with success probabilities $q_{i+1}, \ldots, q_{n}$. Conditioning on the number of successes, say $l$, out of the $n-i$ trials with success probabilities $q_{i+1}, \ldots, q_{n}$, we get:

$$
\begin{aligned}
f_{i, k}(y)= & \sum_{l=k}^{n-i} v_{l}^{i}\left(q_{i+1}, \ldots, q_{n}\right) \\
& +\sum_{l=0}^{\min (k-1, n-i)} v_{l}^{i}\left(q_{i+1}, \ldots, q_{n}\right) h_{k}(y),
\end{aligned}
$$

where $h_{k}(y)=\sum_{m=k-l}^{i-1}\left(\begin{array}{l}i-1 \\ m\end{array}\right) y^{m}(1-y)^{i-1-m}$. Now, for $l$ satisfying:

$$
1 \leq k-l \leq i-1,
$$

$h_{k}(y)$ is a strictly increasing function of $y$ [3]. Also, it can be checked that $l=\min (k-1, n-i)$, which is one of the indices in the expression in (60), satisfies (61). So $f_{i, k}(y)$ is a strictly increasing function of $y$. Also, note that $f_{i, k}($.$) is a$ continuous function. Thus, $f_{i}(y)$ is a strictly increasing and continuous function of $y$ as well (since by assumptions on $\left\{\gamma_{k}\right\} \gamma_{k}>0$ for some $k$ between 1 and $n-1$ ).

Now, it can be checked from the definition of the function $f_{i}($.$) that:$

$$
f_{i}\left(q_{i+1}\right)=f_{i+1}\left(q_{i+1}\right) .
$$

Also, replacing $i$ with $i+1$ in (12), we get:

$$
f_{i+1}\left(q_{i+1}\right)=g\left(R_{i+1}\right) .
$$

By (62) and (63), we get:

$$
f_{i}\left(q_{i+1}\right)=g\left(R_{i+1}\right) .
$$

Now, as shown above, $f_{i}(y)$ is a continuous and strictly increasing function of $y$. So $f_{i}($.$) is invertible. By (17), \phi($. is unique and is given by:

$$
\phi(x)=f_{i}^{-1}(g(x)), R_{i+1} \leq x<R_{i} .
$$

Also, by (64) and (12), $f_{i}\left(q_{i+1}\right)=g\left(R_{i+1}\right)$ and $f_{i}\left(q_{i}\right)=$ $g\left(R_{i}\right)$. So $f_{i}($.$) is a continuous one-to-one map from the$ compact set $\left[q_{i+1}, q_{i}\right]$ onto $\left[g\left(R_{i+1}\right), g\left(R_{i}\right)\right]$, and hence $f_{i}^{-1}($. is continuous (see Theorem 4.17 in [4]). Also, $g(x)$ in (10) is continuous for all $x \in[\tilde{p}, v)$ since $x \geq \tilde{p}>c$. So from (65), $\phi($.$) is a continuous function on \left[R_{i+1}, R_{i}\right]$, since it is the composition of continuous functions $f_{i}^{-1}$ and $g$ (see Theorem 4.7 in [4]). Also, as shown above, $f_{i}($.$) is strictly increasing;$ so $f_{i}^{-1}($.$) is strictly increasing. Also, using x \geq \tilde{p}>c$, it can be checked from (10) that $g^{\prime}(x)>0$; so $g($.$) is strictly$ increasing. By $(65), \phi($.$) is the composition of the strictly$ increasing functions $f_{i}^{-1}($.$) and g($.$) and hence is strictly$ increasing on $\left[R_{i+1}, R_{i}\right]$. Also, by (5), (6), (12) and (65), $\phi\left(R_{i}\right)=f_{i}^{-1}\left(g\left(R_{i}\right)\right)=q_{i}$.

Thus, the function $\phi($.$) is strictly increasing and continuous$ within each individual interval $\left[R_{i+1}, R_{i}\right]$; also, $\phi\left(R_{i}\right)=q_{i}$, $i=2, \ldots, n$, and hence $\phi($.$) is continuous at the endpoints$ $R_{i}, i=2, \ldots, n$ of these intervals. So $\phi($.$) is strictly increasing$ and continuous on $[\tilde{p}, v)$.

It remains to show that $\phi(\tilde{p})=0$. By definition of the function $f_{i}(),. f_{n}(0)=1-r$. As shown above, $f_{n}($.$) is one-$ to-one. So $f_{n}^{-1}(1-r)=0$. Also, by $(10), g(\tilde{p})=1-r$ and 
by (4), $R_{n+1}=\tilde{p}$. Putting $i=n$ and $x=R_{n+1}=\tilde{p}$ in (65), we get $\phi(\tilde{p})=f_{n}^{-1}(g(\tilde{p}))=f_{n}^{-1}(1-r)=0$.

Proof of Theorem 2: By Lemma 1 and equation (7), the functions $\phi_{i}(),. i=1, \ldots, n$ computed in Section III-B are continuous and non-decreasing on $[\tilde{p}, v]$; also, $\phi_{i}(\tilde{p})=0$ and $\phi_{i}(v)=q_{i}$. This is consistent with the fact that $\phi_{i}($.$) is the$ d.f. of the pseudo-price $p_{i}^{\prime}$ and hence should be non-decreasing and right continuous [5], and $\phi_{i}(v)=q_{i} \psi_{i}(v)=q_{i}$ (see the beginning of Section III).

Now, we have shown in Sections III-A and III-B that (7) is a necessary condition for the functions $\phi_{i}(),. i=1, \ldots, n$ to constitute a NE. We now show sufficiency. Suppose for each $i \in\{1, \ldots, n\}$, primary $i$ uses the strategy $\phi_{i}($.$) in (7). Similar$ to the derivation of (8), the expected payoff that primary $i$ gets at a price $x \in[\tilde{p}, v)$ is:

$$
E\left\{u_{i}\left(x, \psi_{-i}\right)\right\}=(x-c)\left(1-F_{-i}(x)\right) .
$$

Now, for $x \in\left[\tilde{p}, R_{i}\right)$, by (4) and (7), $\phi_{i}(x)=\phi_{1}(x)=\phi(x)$, and hence by Lemma $11, F_{-i}(x)=F_{-1}(x)$. Also note that $\phi($.$) is the solution of (8), (16) and (17). By (8), (66) and the$ fact that $F_{-i}(x)=F_{-1}(x)$, for primary $i$, prices $x \in\left[\tilde{p}, R_{i}\right)$ fetch an expected payoff of $(\tilde{p}-c) r$.

Now let $x \in\left[R_{i}, v\right)$. Note that $R_{i} \leq x<v=R_{1}$. So by (7), $\phi_{i}(x)=q_{i}$ and $\phi_{1}(x)=\phi(x) \geq \phi\left(R_{i}\right)=q_{i}$. So $\phi_{1}(x) \geq \phi_{i}(x)$. Hence, by Lemma $11, F_{-1}(x) \leq F_{-i}(x)$, which by (8) and (66) implies $E\left\{u_{i}\left(x, \psi_{-i}\right)\right\} \leq(\tilde{p}-c) r$.

Finally, note that a price below $\tilde{p}$ fetches a payoff of less than $(\tilde{p}-c) r$ for primary $i$. So each price in $\left[\tilde{p}, R_{i}\right)$ is a best response for primary $i$; also, by (7), it randomizes over prices only in this range under $\phi_{i}($.$) . So \phi_{i}($.$) is a best response.$ Thus, the functions $\phi_{i}(),. i=1, \ldots, n$ constitute a NE.

\section{Proof of Theorem 3}

We first outline the proof in Section $\mathrm{C} 1$ and then provide the details in Section C2.

1) Proof Outline: We first state a property of mean valid graphs for later use.

Lemma 16: Let $G=(V, E)$ be a graph that satisfies Condition 1 in Definition 3. Suppose $I \in \mathscr{I}$ contains $m_{j}(I)$ nodes from $I_{j}, j=1, \ldots, d$. $G$ is mean valid if and only if:

$$
\sum_{j=1}^{d} \frac{m_{j}(I)}{M_{j}} \leq 1 \forall I \in \mathscr{I}
$$

Proof: Suppose $G$ is mean valid. Fix an $I \in \mathscr{I}$. Let

$$
\mathbf{1}_{I}\left(a_{j, l}\right)= \begin{cases}1, & \text { if } a_{j, l} \in I \\ 0, & \text { else }\end{cases}
$$

Consider a distribution $\left\{\alpha_{j, l}: j=1, \ldots, d ; l=1, \ldots, M_{j}\right\}$ in which bandwidth is offered at node $a_{j, l} \in I_{j}$ w.p. $\alpha_{j, l}=$ $\mathbf{1}_{I}\left(a_{j, l}\right)$. This is a valid distribution because it corresponds to the distribution $\left\{\beta(I)=1, \beta\left(I^{\prime}\right)=0 \forall I^{\prime} \in \mathscr{I}, I^{\prime} \neq I\right\}$. Also,

$$
\sum_{l=1}^{M_{j}} \alpha_{j, l}=\sum_{l=1}^{M_{j}} \mathbf{1}_{I}\left(a_{j, l}\right)=m_{j}(I), j=1, \ldots, d
$$

Let $\bar{\alpha}_{j}$ be given by (21). Since the graph is mean valid, (21) holds. Substituting $\sum_{l=1}^{M_{j}} \alpha_{j, l}=m_{j}(I)$ from (68) into (21), we get (67).
To prove the converse, suppose (67) holds. Let $\left\{\alpha_{j, l}: j=\right.$ $\left.1, \ldots, d ; l=1, \ldots, M_{j}\right\}$ be a valid distribution. By definition, there exists a distribution $\{\beta(I): I \in \mathscr{I}\}$ such that:

$$
\alpha_{j, l}=\sum_{I \in \mathscr{I}: a_{j, l} \in I} \beta(I)
$$

which can be written as:

$$
\alpha_{j, l}=\sum_{I \in \mathscr{I}} \beta(I) \mathbf{1}_{I}\left(a_{j, l}\right)
$$

Now,

$$
\begin{aligned}
& \sum_{j=1}^{d}\left(\frac{\sum_{l=1}^{M_{j}} \alpha_{j, l}}{M_{j}}\right) \\
= & \sum_{j=1}^{d} \frac{1}{M_{j}}\left\{\sum_{l=1}^{M_{j}} \sum_{I \in \mathscr{I}} \beta(I) \mathbf{1}_{I}\left(a_{j, l}\right)\right\} \quad \text { by (70)) } \\
= & \sum_{I \in \mathscr{I}} \beta(I)\left\{\sum_{j=1}^{d} \frac{\sum_{l=1}^{M_{j}} \mathbf{1}_{I}\left(a_{j, l}\right)}{M_{j}}\right\} \\
= & \sum_{I \in \mathscr{I}} \beta(I)\left\{\sum_{j=1}^{d} \frac{m_{j}(I)}{M_{j}}\right\} \quad\left(\text { since } \sum_{l=1}^{M_{j}} \mathbf{1}_{I}\left(a_{j, l}\right)=m_{j}(I)\right) \\
\leq & 1 \text { by }(67))
\end{aligned}
$$

So (21) holds and hence $G$ is mean valid.

We now outline the proof of Theorem 3. The proof of part 1 of Theorem 3 is straightforward: let $\left\{v_{1}, \ldots, v_{e}\right\}$ be the nodes of the clique. $I_{j}=\left\{v_{j}\right\}, j=1, \ldots, e$ are disjoint maximal I.S. whose union is $V$. Also, these are the only I.S. in the graph; so (67) holds and the clique is mean valid by Lemma 16.

Next, we state some lemmas, which we prove in Section C2, and use to prove the other parts of Theorem 3 .

Lemma 17: Let $G=(V, E)$ be a mean valid graph, where $V=I_{1} \cup \ldots \cup I_{d}$ and $I_{1}, \ldots, I_{d}$ are disjoint maximal I.S. Let $E^{\prime} \supseteq E$ be any set such that no edge in $E^{\prime}$ is between two nodes in the same I.S. $I_{j}, j \in\{1, \ldots, d\}$. Then the graph $G^{\prime}=\left(V, E^{\prime}\right)$ is mean valid.

Thus, if a graph $G$ is mean valid, then the graph $G^{\prime}$ obtained by adding edges in any fashion to $G$, while ensuring that $I_{j}$, $j=1, \ldots, d$ continue to be I.S. in $G^{\prime}$, is a mean valid graph as well.

Lemma 18: Suppose for each $i=1, \ldots, N, G^{i}=\left(V^{i}, E^{i}\right)$ is a mean valid graph, where $V^{i}=I_{1}^{i} \cup \ldots \cup I_{d}^{i}, I_{1}^{i}, \ldots, I_{d}^{i}$ are disjoint maximal I.S., and $\left|I_{j}^{i}\right|=M_{j}^{i}, j=1, \ldots, d$. Let $\mathbf{M}^{i}=\left(M_{1}^{i}, \ldots, M_{d}^{i}\right)$. If

$$
\mathbf{M}^{i}=c_{i} \mathbf{M}^{0}, i=1, \ldots, N
$$

for some vector $\mathbf{M}^{0}=\left(M_{1}^{0}, \ldots, M_{d}^{0}\right)$ and positive scalars $c_{1}, \ldots, c_{N}$, then $G=\left(\cup_{i=1}^{N} V^{i}, \cup_{i=1}^{N} E^{i}\right)$ is mean valid.

Lemma 18 says that if $G^{i}, i=1, \ldots, N$ are mean valid graphs, then their union $G$ is a mean valid graph as well provided each of $G^{i}, i=1, \ldots, N$ contains (i) the same number, $d$, of disjoint maximal I.S., and (ii) the same proportion of nodes in the $d$ I.S. $I_{1}^{i}, \ldots, I_{d}^{i}$. Since the union graph $G$ is a disconnected graph with $N$ components, Lemma 18 is not useful by itself to prove that a graph is mean valid. But it can 
be effectively used in conjunction with Lemma 17 to combine a set of $N$ mean valid graphs into a new connected mean valid graph by (i) first considering their union, which is a disconnected graph, (ii) and then adding some edges to it to make it connected.

A useful special case is when each of these $N$ graphs $G^{i}$ is a clique of size $d$ (which is mean valid by Part 1 of Theorem 3) with vertex set $V^{i}=\left\{v_{1}^{i}, \ldots, v_{d}^{i}\right\}$. Note that these graphs satisfy the hypothesis of Lemma 18 with $I_{j}^{i}=\left\{v_{j}^{i}\right\}, M_{j}^{i}=1$, $\forall i, j, \mathbf{M}^{0}=(1, \ldots, 1)$ and $c_{i}=1 \forall i$. This special case can be used to prove the mean validity of several of the graphs mentioned in Theorem 3, as we explain below.

For an integer $m \geq 1$, let $m_{e}$ (respectively, $m_{o}$ ) denote the greatest even (respectively, odd) integer less than or equal to $m$.

We now prove part 2 of Theorem 3. Consider a linear graph $\mathcal{G}_{m}$ with node set $\left\{v_{1}, v_{2}, \ldots, v_{m}\right\}$ as shown in part (a) of Fig. 2. First, let $m$ be even- say $m=2 N$. For $i=1, \ldots, N$, let $G^{i}$ be the clique of size 2 with the node set $V^{i}=\left\{v_{2 i-1}, v_{2 i}\right\}$ and the edge between the two nodes. In the notation of Lemma 18, let $I_{1}^{i}=\left\{v_{2 i-1}\right\}$ and $I_{2}^{i}=\left\{v_{2 i}\right\}$. By Lemma 18, $G=G^{1} \cup G^{2} \cup \ldots \cup G^{N}$ is a mean valid graph with $d=2$ and the disjoint maximal I.S. $I_{1}=\left\{v_{1}, v_{3}, v_{5}, \ldots v_{m_{o}}\right\}$ and $I_{2}=\left\{v_{2}, v_{4}, v_{6}, \ldots, v_{m_{e}}\right\}$. We can obtain $\mathcal{G}_{m}$ by adding the edges $\left(v_{2}, v_{3}\right),\left(v_{4}, v_{5}\right), \ldots,\left(v_{2 N-2}, v_{2 N-1}\right)$ to $G$ as illustrated in part (a) of Fig. 11. Note that no edge is between two nodes in the same I.S. $I_{j}, j \in\{1,2\}$; so the hypothesis of Lemma 17 is satisfied. Hence, $\mathcal{G}_{m}$ is mean valid by Lemma 17. The proof of the fact that $\mathcal{G}_{m}$ is also mean valid for $m$ odd is deferred until Section C2.

Now, we prove part 3 of Theorem 3. Consider $\mathcal{G}_{m, m}$, where $m$ may be odd or even. Let $v_{i j}$ be the node in the $i$ 'th row and $j$ 'th column $i, j \in\{1, \ldots, m\}$ (see part (b) of Fig. 2). We start with a line graph $\mathcal{G}_{m^{2}}$, which is mean valid by part 2 of Theorem 3, and add some edges to obtain $\mathcal{G}_{m, m}$ as shown in Fig. 12. Specifically, let $\mathcal{G}_{m^{2}}$ be the line graph with the set of nodes $\left\{v_{1,1}, v_{1,2}, \ldots, v_{1, m}, v_{2, m}, v_{2, m-1}, \ldots, v_{2,1}, v_{3,1}\right.$, $\left.v_{3,2}, \ldots, v_{3, m}, v_{4, m}, v_{4, m-1}, \ldots\right\}$ and an edge between each pair of consecutive nodes in this order. $\mathcal{G}_{m^{2}}$ is mean valid with $d=2$, and the disjoint maximal I.S. $I_{1}=\left\{v_{11}, v_{13}\right.$, $\left.\ldots, v_{1, m_{o}}, v_{22}, v_{24}, \ldots, v_{2, m_{e}}, v_{31}, v_{33}, \ldots, v_{3, m_{o}}, \ldots\right\}$ and $I_{2}=\left\{v_{12}, v_{14}, \ldots, v_{1, m_{e}}, v_{21}, v_{23}, \ldots, v_{2, m_{o}}, v_{32}, v_{34}, \ldots\right.$, $\left.v_{3, m_{e}}, \ldots\right\} . \mathcal{G}_{m, m}$ can be obtained from $\mathcal{G}_{m^{2}}$ by adding the remaining edges shown dotted in Fig. 12. Note that no edge is between the same I.S. $I_{j}, j=1,2$. So $\mathcal{G}_{m, m}$ is mean valid by Lemma 17.

Next, we prove part 4 of Theorem 3. Consider $\mathcal{H}_{m, m}$ (see part (a) of Fig. 3). As in $\mathcal{G}_{m, m}$, let $v_{i j}$ be the node in the $i$ 'th row and $j$ 'th column. Let $d=4, I_{1}=\left\{v_{11}, v_{13}, v_{15}\right.$, $\left.\ldots, v_{1, m_{o}}, v_{31}, v_{33}, v_{35}, \ldots, v_{3, m_{o}}, \ldots\right\}, I_{2}=\left\{v_{12}, v_{14}, v_{16}\right.$, $\left.\ldots, v_{1, m_{e}}, v_{32}, v_{34}, v_{36}, \ldots, v_{3, m_{e}}, \ldots\right\}, I_{3}=\left\{v_{21}, v_{23}, v_{25}\right.$, $\left.\ldots, v_{2, m_{o}}, v_{41}, v_{43}, v_{45}, \ldots, v_{4, m_{o}}, \ldots\right\}$ and $I_{4}=\left\{v_{22}, v_{24}\right.$, $\left.v_{26}, \ldots, v_{2, m_{e}}, v_{42}, v_{44}, v_{46}, \ldots, v_{4, m_{e}}, \ldots\right\}$ (see part (b) of Fig. 11). Note that $I_{1}, I_{2}, I_{3}$ and $I_{4}$ are disjoint maximal I.S. For $i, j \in\{1, \ldots, m-1\}$, let $C_{i, j}$ be the clique consisting of the nodes $\left\{v_{i, j}, v_{i, j+1}, v_{i+1, j}, v_{i+1, j+1}\right\}$ and the edges among them (see Fig. 13). First, let $m$ be even. The proof that $\mathcal{H}_{m, m}$ is mean valid is similar to the above proof of mean validity of $\mathcal{G}_{m}$ with $m$ even: we can obtain $\mathcal{H}_{m, m}$ by considering the union of the cliques $C_{i, j}, i, j \in\{1,3,5, \ldots, m-1\}$, which is a mean valid graph by Lemma 18 , and then adding the remaining edges as illustrated in part (b) of Fig. 11. Note that no edge is between two nodes in the same I.S. $I_{j}, j \in\{1,2,3,4\}$; so the hypothesis of Lemma 17 is satisfied. Hence, $\mathcal{H}_{m, m}$ is mean valid by Lemma 17 . The proof of the fact that $\mathcal{H}_{m, m}$ is also mean valid for $m$ odd is deferred until Section C2.

The proof of part 5 of Theorem 3 is similar to that of part 4: we outline the differences. For $i, j, l \in\{1, \ldots, m\}$, let $v_{i j l}$ be the node in the $i$ 'th row, $j$ 'th column and $l^{\prime}$ th level (in the direction normal to the plane of the paper). The node set of $\mathcal{T}_{m, m, m}$ can be partitioned into 8 disjoint maximal I.S. $I_{1}, \ldots, I_{8}$ similar to $I_{1}, \ldots, I_{4}$ for $\mathcal{H}_{m, m}$ (see Fig. 4). Also, cliques $C_{i j l}, i, j, l \in\{1, \ldots, m-1\}$ of size 8 each can be defined similar to the cliques $C_{i j}$ for $\mathcal{H}_{m, m}$. For $m$ even, we can obtain $\mathcal{T}_{m, m, m}$ by considering the union of the cliques $C_{i j l}, i, j, l \in\{1,3,5, \ldots, m-1\}$ and then adding the remaining edges. The fact that $\mathcal{T}_{m, m, m}$ is mean valid then follows from Lemmas 18 and 17. The proof of the fact that $\mathcal{T}_{m, m, m}$ is also mean valid for $m$ odd is outlined in Section C2.

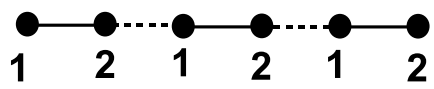

(a)

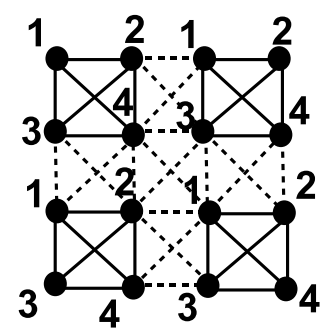

(b)

Fig. 11. Part (a) (respectively, part (b)) shows the construction of $\mathcal{G}_{6}$ (respectively, $\mathcal{H}_{4,4}$ ) from 3 (respectively, 4) cliques of size 2 (respectively, 4) each. The solid edges constitute the cliques $G^{1}, G^{2}, G^{3}$ (respectively, $C_{1,1}, C_{1,3}, C_{3,1}$ and $\left.C_{3,3}\right)$ and the dotted edges are those that are added later. The numbers next to the nodes shows the I.S. they are in, i.e., a node labeled $j$ is in I.S. $I_{j}$, where $j \in\{1,2\}$ (respectively, $j \in\{1,2,3,4\}$ ). Note that no edge is between two nodes in the same I.S. $I_{j}$; so the hypothesis of Lemma 17 is satisfied.

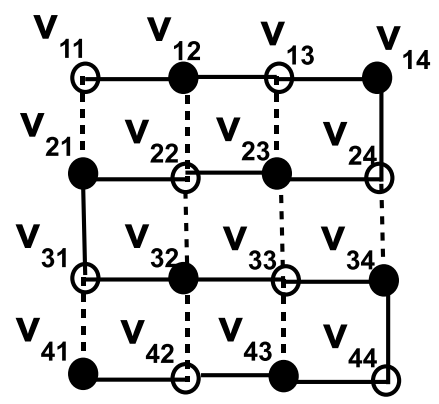

Fig. 12. The figure shows the construction of the grid graph $\mathcal{G}_{m, m}$ from the line graph $\mathcal{G}_{m^{2}}$ for $m=4$. The solid edges constitute $\mathcal{G}_{m^{2}}$ and the dotted edges are later added to obtain $\mathcal{G}_{m, m}$. The un-darkened and darkened nodes constitute $I_{1}$ and $I_{2}$ respectively in both $\mathcal{G}_{m^{2}}$ and $\mathcal{G}_{m, m}$. Note that no edge is between a node in $I_{1}$ and a node in $I_{2}$, so the hypothesis of Lemma 17 is satisfied. 
We now prove part 6 of Theorem 3. Consider a cellular network as shown in Fig. 5, whose conflict graph is shown in Fig. 6. The nodes in the graph can be partitioned into three disjoint maximal I.S. $I_{1}, I_{2}$ and $I_{3}$ as shown in Fig. 6. We consider this conflict graph with the following assumption, which eliminates problems arising due to boundary effects.

Assumption 1: There are an even number of rows of nodes, each containing $3 \delta$ nodes, for some integer $\delta \geq 1$.

Under this assumption, as illustrated in Fig. 6, the graph can be obtained by considering the union of $r \eta$ disjoint cliques of size 3 each, which is a mean valid graph by Lemma 18 , and then adding some edges. Note that no edge is between two nodes in the same I.S. $I_{j}, j \in\{1,2,3\}$ (see Fig. 6); so the hypothesis of Lemma 17 is satisfied. Hence, the graph is mean valid by Lemma 17 .

Note that the above proof goes through if the graph can be partitioned into cliques of size 3 even if Assumption 1 is not satisfied. If the graph cannot be partitioned into cliques of size 3 , then the analysis is more complicated because of boundary effects. We omit this analysis for brevity.

2) Details of Proofs: We now provide the details of the proofs in Section C1.

Proof of Lemma 17: Since no edge in $E^{\prime}$ is between two nodes in the same I.S. $I_{j}$, it follows that in $G^{\prime}, I_{1}, \ldots, I_{d}$ are disjoint maximal I.S. whose union is $V$. Using the notation in Definition 3, let $\left\{\alpha_{j, l}: j=1, \ldots, d ; l=1, \ldots, M_{j}\right\}$ be a valid distribution in $G^{\prime}$. We will show that (21) holds. Then it will follow from Definition 3 that $G^{\prime}$ is mean valid.

Let $\mathscr{I}_{G^{\prime}}$ (respectively, $\mathscr{I}_{G}$ ) be the set of I.S. in $G^{\prime}$ (respectively, $G$ ). Since $E \subset E^{\prime}$, each I.S. in $G^{\prime}$ is an I.S. in $G$ as well, i.e. $\mathscr{I}_{G^{\prime}} \subset \mathscr{I}_{G}$.

Now, since the distribution $\left\{\alpha_{j, l}\right\}$ is valid in $G^{\prime}$, by definition, there exists a distribution $\left\{\beta^{\prime}(I): I \in \mathscr{I}_{G^{\prime}}\right\}$ such that

$$
\alpha_{v}=\sum_{I \in \mathscr{I}_{G^{\prime}}: v \in I} \beta^{\prime}(I) \forall v \in V .
$$

Define a distribution on $\mathscr{I}_{G}$ as follows:

$$
\beta(I)= \begin{cases}\beta^{\prime}(I) & \text { if } I \in \mathscr{I}_{G^{\prime}} \\ 0 & \text { if } I \in \mathscr{I}_{G} \backslash \mathscr{I}_{G^{\prime}}\end{cases}
$$

By (72) and (73):

$$
\alpha_{v}=\sum_{I \in \mathscr{I}_{G}: v \in I} \beta(I) \forall v \in V .
$$

So by definition, $\left\{\alpha_{i, j}\right\}$ is a valid distribution in $G$ as well. Since $G$ is mean valid, (21) holds, which completes the proof.

Proof of Lemma 18: First, note that $\left\{\left(I_{j}^{1} \cup \ldots \cup I_{j}^{N}\right): j=\right.$ $1, \ldots, d\}$ are disjoint maximal I.S. in $G$; so the first condition in Definition 3 is satisfied.

Let $\left\{\alpha_{j, l}^{i}: j=1, \ldots, d ; l=1, \ldots, M_{j}^{i}\right\}$ be a valid distribution in $G^{i}$. Since $G^{i}$ is mean valid:

$$
\sum_{j=1}^{d}\left(\frac{\sum_{l=1}^{M_{j}^{i}} \alpha_{j, l}^{i}}{M_{j}^{i}}\right) \leq 1, \quad i=1, \ldots, N
$$

Now, it is given that:

$$
M_{j}^{i}=c_{i} M_{j}^{0}, \quad i=1, \ldots, N ; j=1, \ldots, d
$$

Adding (76) over $i=1, \ldots, N$ :

$$
M_{j}^{0}\left(c_{1}+\ldots+c_{N}\right)=M_{j}^{1}+\ldots+M_{j}^{N}, j=1, \ldots, d
$$

Multiplying (75) by $c_{i}$, using (76) and adding over $i=$ $1, \ldots, N$, we get:

$$
\sum_{i=1}^{N} \sum_{j=1}^{d}\left(\frac{\sum_{l=1}^{M_{j}^{i}} \alpha_{j, l}^{i}}{M_{j}^{0}}\right) \leq c_{1}+\ldots+c_{N}
$$

Dividing both sides by $c_{1}+\ldots+c_{N}$ and using (77):

$$
\sum_{j=1}^{d}\left(\frac{\sum_{i=1}^{N} \sum_{l=1}^{M_{j}^{i}} \alpha_{j, l}^{i}}{M_{j}^{1}+\ldots+M_{j}^{N}}\right) \leq 1
$$

So $G$ satisfies the second condition in Definition 3 as well and hence is mean valid.

Proof of part 2 of Theorem 3: First, we state a property of bipartite graphs. Consider a connected bipartite graph [6] of the form $G=(V, E)$ where $V=A \cup B$ and every edge is between a node in $A$ and a node in $B$. Without loss of generality, suppose $|A| \leq|B|$. In the notation of Definition 3, $d=2, I_{1}=B$ and $I_{2}=A$. Also, a necessary condition for a node distribution $\left\{\alpha_{i}, i \in A ; \gamma_{j}, j \in B\right\}$, under which bandwidth is offered at node $i \in A$ (respectively, $j \in B$ ) w.p. $\alpha_{i}$ (respectively, $\gamma_{j}$ ), to be valid is that

$$
\alpha_{i}+\gamma_{j} \leq 1 \quad \forall(i, j) \in E .
$$

This is because, if $\alpha_{i}+\gamma_{j}>1$ for some $(i, j) \in E$, then with a positive probability bandwidth would be offered at both nodes $i$ and $j$, which are neighbors.

Now, in Section $\mathrm{C} 1$, we showed that $\mathcal{G}_{m}$ is mean valid for even $m$. Now, let $m$ be odd, say $m=2 N-1$ for some integer $N \geq 2$. Consider a valid distribution $\left\{\alpha_{i}: i=1, \ldots, 2 N-1\right\}$, where $\alpha_{i}$ is the probability with which bandwidth is offered at node $v_{i}$. With $I_{1}$ and $I_{2}$ as defined in Section C1, note that $\left|I_{1}\right|=N$ and $\left|I_{2}\right|=N-1$. Let

$$
\bar{\alpha}^{1}=\frac{\alpha_{1}+\alpha_{3}+\ldots+\alpha_{2 N-1}}{N}
$$

and

$$
\bar{\alpha}^{2}=\frac{\alpha_{2}+\alpha_{4}+\ldots+\alpha_{2 N-2}}{N-1}
$$

To show that Condition 2 in Definition 3 is satisfied, we need to show that $\bar{\alpha}^{1}+\bar{\alpha}^{2} \leq 1$, i.e.

$$
\begin{aligned}
& (N-1)\left(\alpha_{1}+\alpha_{3}+\ldots+\alpha_{2 N-1}\right) \\
& \quad+\quad N\left(\alpha_{2}+\alpha_{4}+\ldots+\alpha_{2 N-2}\right) \leq N(N-1)
\end{aligned}
$$

Since $\mathcal{G}_{2 N-1}$ is a bipartite graph and the distribution $\left\{\alpha_{i}\right\}$ is valid, the necessary condition in (78) holds and in this case becomes:

$$
\alpha_{i}+\alpha_{i+1} \leq 1, \quad i=1,2, \ldots, 2 N-2
$$


Now,

$$
\begin{aligned}
\text { LHS of } & (79) \\
= & \left\{(N-1)\left(\alpha_{1}+\alpha_{2}\right)+\left(\alpha_{2}+\alpha_{3}\right)\right\} \\
& +\left\{(N-2)\left(\alpha_{3}+\alpha_{4}\right)+2\left(\alpha_{4}+\alpha_{5}\right)\right\} \\
& +\left\{(N-3)\left(\alpha_{5}+\alpha_{6}\right)+3\left(\alpha_{6}+\alpha_{7}\right)\right\} \\
& +\ldots \\
& +\left\{2\left(\alpha_{2 N-5}+\alpha_{2 N-4}\right)+(N-2)\left(\alpha_{2 N-4}+\alpha_{2 N-3}\right)\right\} \\
& +\left\{\left(\alpha_{2 N-3}+\alpha_{2 N-2}\right)+(N-1)\left(\alpha_{2 N-2}+\alpha_{2 N-1}\right)\right\} \\
\leq \quad & \{(N-1)+1\}+\{(N-2)+2\}+\ldots \\
& +\{2+(N-2)\}+\{1+(N-1)\}(\text { by }(80)) \\
= & N(N-1)
\end{aligned}
$$

which proves (79) and the result follows.

Proof of part 4 of Theorem 3: In Section C1, we showed that $\mathcal{H}_{m, m}$ is mean valid for even $m$. Now, let $m$ be odd. With $I_{1}, I_{2}, I_{3}$ and $I_{4}$ as defined in Section $\mathrm{C} 1$, it is easy to check that $\left|I_{1}\right|=\left(\frac{m+1}{2}\right)^{2},\left|I_{2}\right|=\frac{m^{2}-1}{4},\left|I_{3}\right|=\frac{m^{2}-1}{4}$ and $\left|I_{4}\right|=\left(\frac{m-1}{2}\right)^{2}$.

Consider a valid distribution $\left\{\alpha_{z}: z \in V\right\}$, where $\alpha_{z}$ is the probability with which bandwidth is offered at node $z$. We now show that the graph is mean valid by showing that (21) holds, which in this case becomes:

$$
\begin{array}{r}
(m-1)^{2}\left(\sum_{z \in I_{1}} \alpha_{z}\right)+\left(m^{2}-1\right)\left(\sum_{z \in I_{2}} \alpha_{z}\right)+\left(m^{2}-1\right)\left(\sum_{z \in I_{3}} \alpha_{z}\right) \\
+(m+1)^{2}\left(\sum_{z \in I_{4}} \alpha_{z}\right) \leq \frac{\left(m^{2}-1\right)^{2}}{4} .
\end{array}
$$

Consider cliques $C_{i, j}, i, j \in\{0, \ldots, m\}$. For $i, j \in$ $\{1, \ldots, m-1\}, C_{i, j}$ is as defined in Section C1. For $i$ or $j$ (or both) equal to 0 or $m$, let $C_{i, j}$ be "dummy cliques", defined for convenience (see Fig. 13). For $i, j \in\{0, \ldots, m\}$ :

$$
\sum_{z \in C_{i j}} \alpha_{z} \leq 1
$$

because, if not, then bandwidth would be offered simultaneously at two or more of the nodes in $C_{i j}$ (which are neighbors) with a positive probability. For $i \in\{0, \ldots, m\}$, let:

$$
e_{i}= \begin{cases}m-i, & i \text { odd } \\ i, & i \text { even }\end{cases}
$$

For $i, j \in\{0, \ldots, m\}$, let

$$
f_{i j}=e_{i} e_{j}
$$

Note that by definition of the cliques $\left\{C_{i, j}\right\}$, node $v_{i j}$ belongs to each of the cliques $C_{i-1, j-1}, C_{i-1, j}, C_{i, j-1}$ and $C_{i, j}$ as shown in Fig. 14. So multiplying (82) by $f_{i j}$ and adding over $i, j \in\{0,1, \ldots, m\}$ gives:

$$
\sum_{z \in V} g_{z} \alpha_{z} \leq g_{0}
$$

where,

$$
g_{v_{i j}}=f_{i-1, j-1}+f_{i-1, j}+f_{i, j-1}+f_{i j}
$$

and

$$
\begin{aligned}
g_{0} & =\sum_{i=0}^{m} \sum_{j=0}^{m} f_{i, j}=\sum_{i=0}^{m} \sum_{j=0}^{m} e_{i} e_{j}=\left(\sum_{i=0}^{m} e_{i}\right)^{2} \\
& =\left(\sum_{i=0, i \text { odd }}^{m}(m-i)+\sum_{i=0, i \text { even }}^{m} i\right)^{2}=\frac{\left(m^{2}-1\right)_{(87)}^{2}}{4}
\end{aligned}
$$

We will show below that

$$
g_{z}= \begin{cases}(m-1)^{2}, & z \in I_{1} \\ \left(m^{2}-1\right), & z \in I_{2} \text { or } z \in I_{3} \\ (m+1)^{2}, & z \in I_{4}\end{cases}
$$

Note that (81) follows from (85), (87) and (88), which shows that $\mathcal{H}_{m, m}$ is mean valid.

Now we show (88). By definition of the I.S. $I_{1}, I_{2}, I_{3}$ and $I_{4}$ (see Section C1), for $v_{i j} \in I_{1}, i$ and $j$ are odd, for $v_{i j} \in I_{2}$, $i$ is odd and $j$ is even, for $v_{i j} \in I_{3}, i$ is even and $j$ is odd and for $v_{i j} \in I_{4}, i$ and $j$ are even. So for $v_{i j} \in I_{1}$, by (83), (84) and (86):

$$
\begin{aligned}
g_{v_{i j}}= & (i-1)(j-1)+(i-1)(m-j)+(m-i)(j-1) \\
& +(m-i)(m-j) \\
= & (m-1)^{2}
\end{aligned}
$$

Similarly, for $v_{i j} \in I_{2}$ :

$g_{v_{i j}}=(i-1)(m-j+1)+(i-1) j+(m-i)(m-j+1)$ $+(m-i) j$

$=m^{2}-1$

For $v_{i j} \in I_{3}, g_{v_{i j}}=m^{2}-1$ by symmetry with the case $v_{i j} \in I_{2}$. For $v_{i j} \in I_{4}$ :

$$
\begin{aligned}
g_{v_{i j}}= & (m-i+1)(m-j+1)+(m-i+1) j \\
& +i(m-j+1)+i j \\
= & (m+1)^{2}
\end{aligned}
$$

Thus, we have shown (88), which completes the proof.

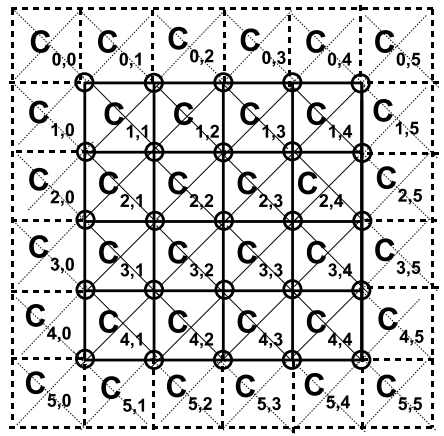

Fig. 13. The figure shows the cliques in $\mathcal{H}_{5,5}$. The cliques with dotted outlines are the dummy cliques.

Proof of part 5 of Theorem 3: In Section C1, we considered the case $m$ even. The proof of the fact that $\mathcal{T}_{m, m, m}$ is mean valid for $m$ odd is similar to that for $\mathcal{H}_{m, m}$ with $m$ odd; we outline the differences. We define the cliques $C_{i j l}$, $i, j, l \in\{0,1, \ldots, m\}$, similar to $C_{i j}$ for the case $\mathcal{H}_{m, m}$. 


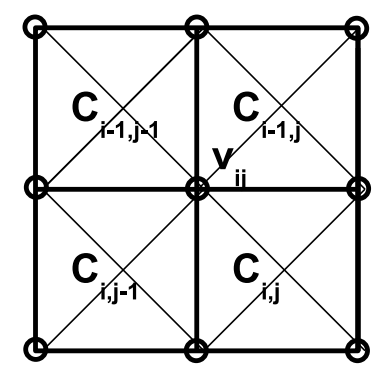

Fig. 14. The node $v_{i j}$ and the cliques $C_{i-1, j-1}, C_{i-1, j}, C_{i, j-1}$ and $C_{i, j}$.

Consider a valid distribution $\left\{\alpha_{z}: z \in V\right\}$. Then similar to (82), we get:

$$
\sum_{z \in C_{i j l}} \alpha_{z} \leq 1
$$

Let $e_{i}$ be as in (83) and $f_{i j l}=e_{i} e_{j} e_{l}, i, j, l \in$ $\{0, \ldots, m\}$. Multiplying (89) by $f_{i j l}$ and adding over $i, j, l \in$ $\{0,1, \ldots, m\}$, we get (85) for some numbers $\left\{g_{z}: z \in V\right\}$ and $g_{0}$. Now, node $v_{i j l}$ is at the center of the cliques $C_{i-1, j-1, l-1}$, $C_{i-1, j-1, l}, C_{i-1, j, l-1}, C_{i-1, j, l}, C_{i, j-1, l-1}, C_{i, j-1, l}, C_{i, j, l-1}$, and $C_{i, j, l}$. Using this fact, $g_{v_{i j l}}$ for $v_{i j l}$ in each of $I_{1}, \ldots, I_{8}$ can be computed similar to the derivation of (88). Also, $g_{0}$ can be calculated similar to (87). Substituting these values of $\left\{g_{z}: z \in V\right\}$ and $g_{0}$ into (85), we get (21) for $\mathcal{T}_{m, m, m}$ and thereby the mean validity follows from Definition 3 .

\section{Proofs of results in Section IV-C}

We first provide the intuition and an outline of the proofs in Section D1 and then provide the details in Section D2.

1) Intuition and Proof Outline: Let the function $w_{1}($.$) be$ as defined in Section IV-C. We will later use the following property, which is quite intuitive:

Lemma 19: $w_{1}(\alpha)$ is a strictly increasing function of $\alpha$ on $[0,1]$.

Proof: Let $0 \leq \alpha<\alpha^{\prime} \leq 1$. It suffices to show that $w_{1}(\alpha)<w_{1}\left(\alpha^{\prime}\right)$.

Let $Y_{i}, i=2, \ldots, n$ be independent Bernoulli random variables and let $Y_{i}$ have mean $q_{i} \alpha$. Also, let $Z_{i}, i=2, \ldots, n$ be independent Bernoulli random variables that are independent of $Y_{i}, i=2, \ldots, n$ and let $Z_{i}$ have mean $\frac{q_{i} \alpha^{\prime}-q_{i} \alpha}{1-q_{i} \alpha}$.

For $i=2, \ldots, n$, let:

$$
X_{i}= \begin{cases}1, & \text { if } Y_{i}=1 \text { or } Z_{i}=1 \text { (or both) } \\ 0, & \text { else }\end{cases}
$$

$$
\begin{aligned}
P\left(X_{i}=1\right)= & \left.P\left(\left\{Y_{i}=1\right\} \cup\left(Z_{i}=1\right)\right\}\right) \\
= & P\left(Y_{i}=1\right)+P\left(Z_{i}=1\right) \\
& -P\left(\left\{Y_{i}=1\right\} \cap\left\{Z_{i}=1\right\}\right) \\
= & P\left(Y_{i}=1\right)+P\left(Z_{i}=1\right)-P\left(Y_{i}=1\right) P\left(Z_{i}=\right. \\
& \left(\text { since } Y_{i} \text { and } Z_{i} \text { are independent }\right) \\
= & q_{i} \alpha+\frac{q_{i} \alpha^{\prime}-q_{i} \alpha}{1-q_{i} \alpha}-\left(q_{i} \alpha\right)\left(\frac{q_{i} \alpha^{\prime}-q_{i} \alpha}{1-q_{i} \alpha}\right) \\
= & q_{i} \alpha^{\prime}
\end{aligned}
$$

So $X_{i}$ is Bernoulli with mean $q_{i} \alpha^{\prime}$. Also, since $Y_{i}, i=2, \ldots, n$ and $Z_{i}, i=2, \ldots, n$ are independent, $X_{i}, i=2, \ldots, n$ are independent.

But by (90),

$$
\left\{Y_{i}=1\right\} \subset\left\{X_{i}=1\right\}, i=1, \ldots, n
$$

Also,

$$
\begin{aligned}
P\left\{X_{i}=1, Y_{i}=0\right\} & =P\left(Z_{i}=1, Y_{i}=0\right) \\
& =P\left(Z_{i}=1\right) P\left(Y_{i}=0\right) \\
& =\left(\frac{q_{i} \alpha^{\prime}-q_{i} \alpha}{1-q_{i} \alpha}\right)\left(1-q_{i} \alpha\right) \\
& =q_{i} \alpha^{\prime}-q_{i} \alpha \\
& >0
\end{aligned}
$$

By (91) and (92):

$$
P\left(X_{i}=1\right)>P\left(Y_{i}=1\right) .
$$

Now, let $X=\sum_{i=2}^{n} X_{i}$ and $Y=\sum_{i=2}^{n} Y_{i}$. We interpret $X_{i}$ (respectively, $Y_{i}$ ) as the indicator of the event that primary $i$ offers bandwidth at a node $v$ with node probability $\alpha_{v}=\alpha^{\prime}$ (respectively, $\alpha_{v}=\alpha$ ). So $X$ (respectively, $Y$ ) is the number of primaries who offer bandwidth at node $v$ when $\alpha_{v}=\alpha^{\prime}$ (respectively, $\alpha_{v}=\alpha$ ). By definition of the function $w_{1}($.$) :$

$$
w_{1}\left(\alpha^{\prime}\right)=P(X \geq k)
$$

and

$$
w_{1}(\alpha)=P(Y \geq k) .
$$

By (93), (94), (95) and the facts $X=\sum_{i=2}^{n} X_{i}$ and $Y=$ $\sum_{i=2}^{n} Y_{i}$, it follows that $w_{1}(\alpha)<w_{1}\left(\alpha^{\prime}\right)$.

Now, let the function $W($.$) be as defined in Section IV-C.$ By Lemma 2, and similar to (18) in the single location case, in a NE in class $\mathcal{S}$, if primaries offer bandwidth at a node w.p. $\alpha$ (and play the single-node NE strategy with $q_{1} \alpha, \ldots, q_{n} \alpha$ in place of $q_{1}, \ldots, q_{n}$ respectively at that node), then $W(\alpha)$ is the maximum expected payoff that each primary $i$ can get at that node. In an NE with strategy profile $\left(\psi_{1}, \ldots, \psi_{n}\right)$, if each primary offers bandwidth at node $v \in V$ w.p. $\alpha_{v}$, then the expected payoff of each primary $i$ is given by:

$$
E\left\{u_{i}\left(\psi_{i}, \psi_{-i}\right)\right\}=\sum_{v \in V} \alpha_{v} W\left(\alpha_{v}\right) .
$$

Also, note that if primary $i$ offers bandwidth at I.S. $I^{\prime} \in \mathscr{I}$, its overall expected payoff, denoted by $U_{1}\left(I^{\prime}\right)$, is the sum of the expected payoffs at the nodes in $I^{\prime}$, which, by (22) is given by:

$$
U_{1}\left(I^{\prime}\right)=\sum_{v \in I^{\prime}} W\left(\alpha_{v}\right)=\sum_{v \in I^{\prime}}\left(1-w_{1}\left(\alpha_{v}\right)\right) .
$$

Now, let $G$ be a mean valid graph. Suppose there exists a NE in class $\mathcal{S}$ in which each primary offers bandwidth at node $a_{j, l}$ w.p. $\alpha_{j, l}, j=1, \ldots, d, l=1, \ldots, M_{j}$, where $\left\{\alpha_{j, l}\right\}$ is a valid distribution. Let the corresponding strategy profile be $\left(\psi_{1}, \ldots, \psi_{n}\right)$. In the NE $\left(\psi_{1}, \ldots, \psi_{n}\right)$, by (22), primary $1^{20}$

\footnotetext{
${ }^{20} \mathrm{The}$ arguments in this section are given from the point of view of primary 1 for concreteness; they also hold for any primary $i \in\{2, \ldots, n\}$ since the I.S. distributions of the primaries are symmetric in an NE in class $\mathcal{S}$.
} 
gets an expected payoff of $W\left(\alpha_{j, l}\right)$ at node $a_{j, l}$; also, by (96), its total expected payoff is:

$$
E\left\{u_{1}\left(\psi_{1}, \psi_{-1}\right)\right\}=\sum_{j=1}^{d} \sum_{l=1}^{M_{j}} \alpha_{j, l} W\left(\alpha_{j, l}\right)
$$

We now prove that for each $j, \alpha_{j, l}=\bar{\alpha}_{j} \forall l=1, \ldots, M_{j}$, where $\bar{\alpha}_{j}$ is given by (21). Suppose not. By (22) and Lemma $19, W(\alpha)$ is a strictly decreasing function of $\alpha$; so primary 1 offers bandwidth with a high probability $\alpha_{j, l}$ at nodes $a_{j, l}$ at which it gets a low payoff $W\left(\alpha_{j, l}\right)$. Suppose now, primary 1 unilaterally switches to a strategy $\psi_{0}$, under which it offers bandwidth at each node in $I_{j}, j \in\{1, \ldots, d\}$ w.p. $\bar{\alpha}_{j}$. Note that $\sum_{j=1}^{d} \bar{\alpha}_{j} \leq 1$ by (21); so $\psi_{0}$ is a valid distribution since it corresponds to the I.S. distribution $\left\{\beta\left(I_{j}\right)=\bar{\alpha}_{j}, j \in\{1, \ldots, d\} ; \beta\left(I_{\emptyset}\right)=1-\sum_{j=1}^{d} \bar{\alpha}_{j}, \beta(I)=0\right.$, $\left.I \neq I_{1}, \ldots, I_{d}, I_{\emptyset}\right\}$. We will next show that this unilateral switch to strategy $\psi_{0}$ increases primary 1's expected payoff, which will in turn contradict the fact that $\left(\psi_{1}, \ldots, \psi_{n}\right)$ is a NE.

By (96), the total expected payoff of primary 1 if it plays strategy $\psi_{0}$ is:

$$
E\left\{u_{1}\left(\psi_{0}, \psi_{-1}\right)\right\}=\sum_{j=1}^{d} \sum_{l=1}^{M_{j}} \bar{\alpha}_{j} W\left(\alpha_{j, l}\right)
$$

By (98) and (99):

$$
\begin{aligned}
& E\left\{u_{1}\left(\psi, \psi_{-1}\right)\right\}-E\left\{u_{1}\left(\psi_{0}, \psi_{-1}\right)\right\} \\
= & \left.\sum_{j=1}^{d}\left(\sum_{l=1}^{M_{j}} \alpha_{j, l} W\left(\alpha_{j, l}\right)-\bar{\alpha}_{j}\left(\sum_{l=1}^{M_{j}} W\left(\alpha_{j, l}\right)\right)\right) 100\right)
\end{aligned}
$$

Now, we have the following algebraic fact, proved in Section D2:

Lemma 20: Let $N \geq 2$ be an integer, $\alpha_{1}, \ldots, \alpha_{N}$ be real numbers and $\bar{\alpha}=\frac{\sum_{i=1}^{N} \alpha_{i}}{N}$. Let $f(x)$ be any strictly decreasing function of $x$. Then:

$$
\left(\sum_{i=1}^{N} \alpha_{i} f\left(\alpha_{i}\right)\right) \leq \bar{\alpha}\left(\sum_{i=1}^{N} f\left(\alpha_{i}\right)\right)
$$

with equality iff $\alpha_{1}=\ldots=\alpha_{N}=\bar{\alpha}$.

Intuitively, since $f($.$) is strictly decreasing, in the LHS of$ (101), the terms in which $f\left(\alpha_{i}\right)$ is large are multiplied by small factors $\alpha_{i}$ and vice-versa; on the other hand, all terms $f\left(\alpha_{i}\right)$ on the RHS are multiplied by the same factor $\bar{\alpha}$. So the LHS is smaller.

Now, as mentioned above, $f(\alpha)=W(\alpha)=1-w_{1}(\alpha)$ is a strictly decreasing function of $\alpha$. So by Lemma 20, the expression in (100) is $<0$, since we have assumed that for at least one value of $j, \alpha_{j, 1}, \ldots, \alpha_{j, M_{j}}$ are not all equal. This contradicts the fact that $\left(\psi_{1}, \ldots, \psi_{n}\right)$ is a NE. Thus, $\alpha_{j, l}=$ $\bar{\alpha}_{j}, l=1, \ldots, M_{j}$.

Now, suppose $\sum_{j=1}^{d} \bar{\alpha}_{j}<1$. Then primary 1 can unilaterally offer bandwidth at each node in $I_{d}$ with probability $1-\sum_{j=1}^{d-1} \bar{\alpha}_{j}>\bar{\alpha}_{d}$ instead of $\bar{\alpha}_{d}$ and increase its payoff. This contradicts the fact that the distribution is a NE. So we must have $\sum_{j=1}^{d} \bar{\alpha}_{j}=1$. Thus, we have shown:
Lemma 21: In a mean valid graph, under every NE in class $\mathcal{S}$, each primary offers bandwidth at each node in $I_{j}$ w.p. $t_{j}, j \in\{1, \ldots, d\}$, for some $t_{j} \geq 0, j=1, \ldots, d$, where $\sum_{j=1}^{d} t_{j}=1$.

The following result, proved in Section D2, provides necessary conditions for a distribution $\left\{t_{j}: j=1, \ldots, d\right\}$ as in Lemma 21 to constitute a NE in class $\mathcal{S}$.

Lemma 22: If a distribution $\left\{t_{j}: j=1, \ldots, d\right\}$ as in Lemma 21 constitutes a NE in class $\mathcal{S}$, then $I_{1}, \ldots, I_{d^{\prime}}$ are best responses and $I_{d^{\prime}+1}, \ldots, I_{d}$ are not, for some integer $d^{\prime} \in\{1, \ldots, d\}$. Also, each $I \in \mathscr{I}$ containing a node from $I_{j}$ for some $j>d^{\prime}$ is not a best response. Hence (23) holds.

Recall that by (20), $I_{1}, \ldots, I_{d}$ are in decreasing order of size. So Lemma 22 says that primaries do not choose I.S. smaller than a certain size (out of $I_{1}, \ldots, I_{d}$ ).

Now, consider a NE in class $\mathcal{S}$ with $\left\{t_{j}: j=1, \ldots, d\right\}$ as in Lemma 21. The expected payoff of primary 1 if it offers bandwidth at $I_{j}$ is the sum of the expected payoffs at the nodes in $I_{j}$, which, by (22), is given by:

$U_{1}\left(I_{j}\right)=\sum_{v \in I_{j}} W\left(\alpha_{v}\right)=\sum_{v \in I_{j}} W\left(t_{j}\right)=\left|I_{j}\right| W\left(t_{j}\right)=M_{j} W\left(t_{j}\right)$.

By Lemma $22, I_{1}, \ldots, I_{d^{\prime}}$ are best responses and $I_{d^{\prime}+1}$ is not. So $U_{1}\left(I_{1}\right)=\ldots=U_{1}\left(I_{d^{\prime}}\right)>U_{1}\left(I_{d^{\prime}+1}\right)$. Substituting (102), and using (23) and the fact that $W(0)=1-w_{1}(0)=r$, we get (24).

Thus, we have shown the following:

Lemma 23: A distribution $\left\{t_{j}: j=1, \ldots, d\right\}$ as in Lemma 21 that constitutes a NE in class $\mathcal{S}$ must satisfy (23) and (24) for some integer $d^{\prime} \in\{1, \ldots, d\}$.

Lemma 23 provides necessary conditions for a distribution $\left\{t_{j}: j=1, \ldots, d\right\}$ to constitute a NE in class $\mathcal{S}$. The following lemma shows that these conditions are sufficient as well.

Lemma 24: Let $1 \leq d^{\prime} \leq d$ and $t_{1}, \ldots, t_{d}$ be a probability distribution such that (23) and (24) hold. Then the strategy profile in which every primary offers bandwidth at each node in $I_{j}$ w.p. $t_{j}, j \in\{1, \ldots, d\}$, is a NE in class $\mathcal{S}$.

The proof of Lemma 24 (see Section D2) is based on the fact that the graph, being mean valid, satisfies Condition 2 in Definition 3.

The following technical lemma, proved in Section D2, shows the existence and uniqueness of a distribution $\left(t_{1}, \ldots, t_{d}\right)$ satisfying (23) and (24).

Lemma 25: There exists a unique integer $d^{\prime}$ and a unique probability distribution $\left(t_{1}, \ldots, t_{d}\right)$ such that (23) and (24) hold. Also, $t_{1} \geq t_{2} \ldots \geq t_{d}$.

Note that the fact that $t_{1} \geq t_{2} \ldots \geq t_{d}$ is consistent with the intuition that primaries offer bandwidth at the larger I.S. with a larger probability.

Finally, putting together the above discussion, we prove both Theorem 4 and Lemma 3.

Proof of Theorem 4 and Lemma 3: By Lemma 21, under every NE in class $\mathcal{S}$, each primary must offer bandwidth at all the nodes in $I_{j}, j \in\{1, \ldots, d\}$, w.p. $t_{j}$ for some probability distribution $\left(t_{1}, \ldots, t_{d}\right)$. Also, by Lemma 23, (23) and (24) hold for this distribution. By Lemma 25, there exists a unique distribution $\left(t_{1}, \ldots, t_{d}\right)$ satisfying (23) and (24). Finally, by 
Lemma 24 , the strategy profile where each primary uses this distribution is a NE in class $\mathcal{S}$. The result follows.

Thus, every mean valid graph has a unique NE in class $\mathcal{S}$, which can be explicitly computed by solving the system of equations (23) and (24).

2) Details of Proofs: Let $W(\alpha)$ be as in (22). We will use the following result throughout.

Lemma 26: (i) For $0 \leq \alpha \leq 1,0 \leq W(\alpha) \leq r$, (ii) $W(0)=$ $r$, and (iii) $W(\alpha)$ is strictly decreasing in $\alpha$.

Lemma 26 follows from (22), the fact that $w_{1}(0)=1-r$ and Lemma 19.

The following lemma is used in the proof of Lemma 20.

Lemma 27: Let $N \geq 2$ be an integer and $\alpha_{1}, \ldots, \alpha_{N}, f_{1}, \ldots, f_{N}$ be real numbers. Then:

$N\left(\sum_{i=1}^{N} \alpha_{i} f_{i}\right)-\left(\sum_{i=1}^{N} \alpha_{i}\right)\left(\sum_{i=1}^{N} f_{i}\right)=\sum_{1 \leq i<j \leq N}\left(\alpha_{j}-\alpha_{i}\right)\left(f_{j}-f_{i}\right)$

Proof: We prove the result by induction. For $N=2$ :

$$
\begin{aligned}
\text { LHS } & =2\left(\alpha_{1} f_{1}+\alpha_{2} f_{2}\right)-\left(\alpha_{1}+\alpha_{2}\right)\left(f_{1}+f_{2}\right) \\
& =\left(\alpha_{2}-\alpha_{1}\right)\left(f_{2}-f_{1}\right) \\
& =\text { RHS }
\end{aligned}
$$

Suppose the result is true for $N$. For $N+1$ :

$$
\begin{aligned}
L H S= & (N+1)\left(\sum_{i=1}^{N} \alpha_{i} f_{i}+\alpha_{N+1} f_{N+1}\right)- \\
& \left(\sum_{i=1}^{N} \alpha_{i}+\alpha_{N+1}\right)\left(\sum_{i=1}^{N} f_{i}+f_{N+1}\right) \\
= & \left\{N\left(\sum_{i=1}^{N} \alpha_{i} f_{i}\right)-\left(\sum_{i=1}^{N} \alpha_{i}\right)\left(\sum_{i=1}^{N} f_{i}\right)\right\} \\
& +N \alpha_{N+1} f_{N+1}+\sum_{i=1}^{N} \alpha_{i} f_{i}+\alpha_{N+1} f_{N+1} \\
& -\alpha_{N+1}\left(\sum_{i=1}^{N} f_{i}\right)-\left(\sum_{i=1}^{N} \alpha_{i}\right) f_{N+1}-\alpha_{N+1} f_{N+1} \\
= & \sum_{1 \leq i<j \leq N}\left(\alpha_{j}-\alpha_{i}\right)\left(f_{j}-f_{i}\right) \\
+ & \sum_{i=1}^{N}\left(\alpha_{N+1} f_{N+1}+\alpha_{i} f_{i}-\alpha_{N+1} f_{i}-\alpha_{i} f_{N+1}\right)
\end{aligned}
$$

(by induction hypothesis and collecting terms)

$$
=R H S
$$

The result follows by induction.

Proof of Lemma 20: By symmetry, we can assume WLOG that $\alpha_{1} \leq \alpha_{2} \ldots \leq \alpha_{N}$. Since $f($.$) is strictly$ decreasing, $f\left(\alpha_{1}\right) \geq f\left(\alpha_{2}\right) \geq \ldots \geq f\left(\alpha_{N}\right)$. Now:

$$
\begin{aligned}
& \left(\sum_{i=1}^{N} \alpha_{i} f\left(\alpha_{i}\right)\right)-\bar{\alpha}\left(\sum_{i=1}^{N} f\left(\alpha_{i}\right)\right) \\
& \quad=\frac{1}{N}\left(N\left(\sum_{i=1}^{N} \alpha_{i} f\left(\alpha_{i}\right)\right)-\left(\sum_{i=1}^{N} \alpha_{i}\right)\left(\sum_{i=1}^{N} f\left(\alpha_{i}\right)\right)\right) \\
& \left.\left.\quad=\frac{1}{N} \sum_{1 \leq i<j \leq N}\left(\alpha_{j}-\alpha_{i}\right)\left(f\left(\alpha_{j}\right)-f\left(\alpha_{i}\right)\right) \text { (by (10Bi) }\right) 4\right)
\end{aligned}
$$

For $i<j, \alpha_{i} \leq \alpha_{j}$ and $f\left(\alpha_{i}\right) \geq f\left(\alpha_{j}\right)$. So each term in (104) is $\leq 0$. Hence, the expression in (104) is 0 iff each term is 0 , which happens iff $\alpha_{1}=\ldots=\alpha_{N}=\bar{\alpha}$.

Proof of Lemma 22: Let

$$
\begin{aligned}
U^{*} & =\max \left\{U_{1}\left(I_{j}\right): j \in\{1, \ldots, d\}\right\} \\
& =\max \left\{M_{j} W\left(t_{j}\right): j \in\{1, \ldots, d\}\right\} \text { (by (102)) }
\end{aligned}
$$

and $B=\left\{j \in\{1, \ldots, d\}: M_{j} W\left(t_{j}\right)=U^{*}\right\}$. Note that $B$ is the set of indices of the I.S. out of $I_{1}, \ldots, I_{d}$ that yield the highest payoff and $U^{*}$ is the value of that payoff.

By definition of $B$ :

$$
\begin{gathered}
W\left(t_{j}\right)=\frac{U^{*}}{M_{j}}, \forall j \in B \\
W\left(t_{j}\right)<\frac{U^{*}}{M_{j}}, \quad \forall j \notin B .
\end{gathered}
$$

Let $I$ be any I.S. containing $m_{j}(I)$ nodes from $I_{j}, j=$ $1, \ldots, d$. By (97):

$$
\begin{aligned}
U_{1}(I) & =\sum_{j=1}^{d} m_{j}(I) W\left(t_{j}\right) \\
& \leq \sum_{j=1}^{d} m_{j}(I)\left(\frac{U^{*}}{M_{j}}\right) \\
& \leq U^{*}(\text { by }(67))
\end{aligned}
$$

(by (105) and (106)(107)

So $\max _{I \in \mathscr{I}} U_{1}(I) \leq U^{*}$, and since $U_{1}\left(I_{j}\right)=U^{*}, j \in B$, each $I_{j}, j \in B$, is a best response. Now, for $I$ as defined above, suppose $m_{j}(I) \geq 1$ for some $j \notin B$. Then the inequality in (107) is strict. So $U_{1}(I)<U^{*}$ and $I$ is not a best response. Thus, each $I \in \mathscr{I}$ containing a node from $I_{j}$ for some $j \notin B$ is not a best response. In particular, $\forall j \notin B, I_{j}$ is not a best response and, since primaries offer bandwidth at $I_{j}$ w.p. $t_{j}$ in the above NE, $t_{j}=0$ for all $j \notin B$.

It now suffices to show that $B=\left\{1, \ldots, d^{\prime}\right\}$ for some $1 \leq d^{\prime} \leq d$. Suppose not. Then there exist $j, l \in\{1, \ldots, d\}$ such that $j<l, j \notin B$ and $l \in B$. Since $j \notin B, t_{j}=0$ by the previous paragraph. Now, by (97):

$$
\begin{aligned}
U_{1}\left(I_{j}\right) & =M_{j} W\left(t_{j}\right) \\
& =M_{j} r(\text { by part (ii) of Lemma 26) } \\
& \geq M_{l} r(\text { by }(20), \text { since } j<l) \\
& \geq M_{l} W\left(t_{l}\right) \quad(\text { by part (i) of Lemma 26) } \\
& =U^{*}
\end{aligned}
$$

So $I_{j}$ is a best response, which is a contradiction since $j \notin B$. 
Proof of Lemma 24: Suppose primaries $2, \ldots, n$ use the strategy $\psi$, under which bandwidth is offered at the nodes in $I_{j}$ w.p. $t_{j}, j=1, \ldots, d$. By (23) and part (ii) of Lemma 26, $W\left(t_{j}\right)=r, j>d^{\prime}$. So by (97), the payoff of primary 1 if it plays I.S. $I_{j}, j \in\left\{1, \ldots, d^{\prime}\right\}$ (resp., $j \in\left\{d^{\prime}+1, \ldots, d\right\}$ ) is $U_{1}\left(I_{j}\right)=M_{j} W\left(t_{j}\right)$ (resp., $U_{1}\left(I_{j}\right)=M_{j} r$ ). Hence, by (24) and (20), for some $U^{*}$,

$$
U^{*}=U_{1}\left(I_{1}\right)=\ldots=U_{1}\left(I_{d^{\prime}}\right)>U_{1}\left(I_{d^{\prime}+1}\right) \geq \ldots \geq U_{1}\left(I_{d}\right) .
$$

The maximum payoff that primary 1 can get at a node $v \in I_{j}$, $j \in\left\{1, \ldots, d^{\prime}\right\}$ equals

$$
W\left(t_{j}\right)=\frac{U_{1}\left(I_{j}\right)}{M_{j}}=\frac{U^{*}}{M_{j}} .
$$

Now, for $j>d^{\prime}, M_{j} r=U_{1}\left(I_{j}\right)<U^{*}$. So the maximum payoff that primary 1 can get at a node $v \in I_{j}, j>d^{\prime}$ is

$$
r<\frac{U^{*}}{M_{j}} .
$$

Now, let $I$ be an I.S. containing $m_{j}(I)$ nodes from $I_{j}, j=$ $1, \ldots, d$. By (108) and (109):

$$
\begin{aligned}
U_{1}(I) & \leq U^{*}\left(\sum_{j=1}^{d} \frac{m_{j}(I)}{M_{j}}\right) \\
& \leq U^{*}(\text { by }(67))
\end{aligned}
$$

Since $U_{1}\left(I_{1}\right)=\ldots=U_{1}\left(I_{d^{\prime}}\right)=U^{*}, I_{1}, \ldots, I_{d^{\prime}}$ are best responses. Under the strategy $\psi$, primary 1 can only play $I_{1}, \ldots, I_{d^{\prime}}$ with positive probability; hence, $\psi$ is a best response.

Proof of Lemma 25: Existence: For convenience, let $M_{d+1}=0$. For $x \in\left[M_{1} W(1), M_{1} r\right]$ and $j \in\{1, \ldots, d\}$, if $M_{j} r \geq x$, then we show that the equation:

$$
M_{j} W\left(t_{j}\right)=x
$$

has a unique solution $t_{j}(x) \in[0,1]$. Let $h\left(t_{j}\right)=M_{j} W\left(t_{j}\right)$. By part (ii) of Lemma 26, $h(0)=M_{j} r \geq x$. Also,

$$
\begin{aligned}
h(1) & =M_{j} W(1) \\
& \leq M_{1} W(1)(\text { by }(20)) \\
& \leq x
\end{aligned}
$$

Also, since $w_{1}($.$) is a continuous function and by (22), h\left(t_{j}\right)$ is a continuous function of $t_{j}$. So by the intermediate value theorem [4], $h\left(t_{j}\right)=x$ has a solution in [0,1]. By part (iii) of Lemma $26, h\left(t_{j}\right)$ is a strictly decreasing function of $t_{j}$; so this solution, say $t_{j}(x)$, is unique. For $x=M_{j} r, t_{j}=0$ satisfies (111) by part (ii) of Lemma 26. So $t_{j}\left(M_{j} r\right)=0$.

Since $h\left(t_{j}\right)$ is strictly decreasing on $0 \leq t_{j} \leq 1$, it is invertible. Also, since the inverse of a continuous function is continuous (see Theorem 4.17 in [4]), $h^{-1}(x)$ is continuous. But $x=h\left(t_{j}(x)\right)$. So $t_{j}(x)=h^{-1}(x)$. Thus, $t_{j}(x)$ is continuous in $x$ for $x \leq M_{j} r$. For $x>M_{j} r$, define $t_{j}(x)=0$. As shown above, $t_{j}\left(M_{j} r\right)=0$. So $t_{j}(x)$ is continuous on $\left[M_{1} W(1), M_{1} r\right]$. Let,

$$
T(x)=\sum_{j=1}^{d} t_{j}(x)
$$

As shown above, $h\left(t_{j}\right)$ is strictly decreasing on $0 \leq t_{j} \leq 1$ for $j=1, \ldots, d$. So $t_{j}(x)=h^{-1}(x)$ is strictly decreasing for $x \leq M_{j} r$. Also, by definition, $t_{j}(x)=0$ on $M_{j} r<x \leq M_{1} r$. So by (112), $T(x)$ is strictly decreasing on $\left[M_{1} W(1), M_{1} r\right]$ (note that $t_{1}(x)$ is strictly decreasing on $x \leq M_{1} r$ ). Also, $t_{j}\left(M_{1} r\right)=0, j=1, \ldots, d$. So

$$
T\left(M_{1} r\right)=0 .
$$

Now, for $j=1$ and $x=M_{1} W(1), t_{1}=1$ satisfies (111). So $t_{1}\left(M_{1} W(1)\right)=1$ and hence, by (112):

$$
T\left(M_{1} W(1)\right) \geq 1 .
$$

Now, since each $t_{j}(x), j=1, \ldots, d$, is continuous on $\left[M_{1} W(1), M_{1} r\right]$, so is $T(x)$ by (112). Hence, by (113), (114) and the intermediate value theorem, the equation $T(x)=1$ has a solution $x^{*} \in\left[M_{1} W(1), M_{1} r\right]$, which is unique because $T(x)$ is strictly decreasing. Let $d^{\prime}=\max \left\{j: M_{j} r \geq x^{*}\right\}$. By definition of $t_{j}(x)$, for $j=1, \ldots, d^{\prime}, M_{j} W\left(t_{j}\left(x^{*}\right)\right)=x^{*}$ and for $j>d^{\prime}, M_{j} r<x^{*}$ and hence $t_{j}\left(x^{*}\right)=0$. Thus, $\left(t_{1}\left(x^{*}\right), \ldots, t_{d}\left(x^{*}\right)\right)$ satisfy (23) and (24). Also, by (112), $\sum_{j=1}^{d} t_{j}\left(x^{*}\right)=T\left(x^{*}\right)=1$; so $\left(t_{1}\left(x^{*}\right), \ldots, t_{d}\left(x^{*}\right)\right)$ is a probability distribution. The result follows.

Uniqueness: We now show the uniqueness of $d^{\prime}$ and the distribution $\left(t_{1}, \ldots, t_{d}\right)$ satisfying (23) and (24). Assume, to reach a contradiction, that there exist $e, f \in\{1, \ldots, d\}$ and probability distributions $t=\left(t_{1}, \ldots, t_{d}\right)$ and $s=\left(s_{1}, \ldots, s_{d}\right)$ such that $t_{j}=0$ (respectively, $s_{j}=0$ ) for $j>e$ (respectively, $j>f$ ) and for some $y$ and $z$ :

$$
\begin{gathered}
y=M_{1} W\left(t_{1}\right)=\ldots=M_{e} W\left(t_{e}\right)>M_{e+1} r \\
z=M_{1} W\left(s_{1}\right)=\ldots=M_{f} W\left(s_{f}\right)>M_{f+1} r
\end{gathered}
$$

First, suppose $e=f$. If $y=z$, then by (115) and (116), $M_{j} W\left(t_{j}\right)=M_{j} W\left(s_{j}\right), j=1, \ldots, e$. By part (iii) of Lemma 26, $W($.$) is a one-to-one function; so t_{j}=s_{j}$, $j=1, \ldots, e$. Also, $t_{j}=s_{j}=0, j>e$. So $t=s$.

Now, suppose $z>y$. Then $M_{j} W\left(s_{j}\right)>M_{j} W\left(t_{j}\right), j=$ $1, \ldots, e$. So $W\left(s_{j}\right)>W\left(t_{j}\right)$, and by part (iii) of Lemma 26 , $s_{j}<t_{j}, j=1, \ldots, e$. So $1=\sum_{j=1}^{e} s_{j}<\sum_{j=1}^{e} t_{j}=1$, which is a contradiction. Thus, $z>y$ is not possible. By symmetry, $z<y$ is also not possible.

Now, suppose $e<f$. Then by (115) and (116), $z=$ $M_{e+1} W\left(s_{e+1}\right) \leq M_{e+1} r<y$. So for $j \in\{1, \ldots, e\}$ :

$$
M_{j} W\left(s_{j}\right)=z<y=M_{j} W\left(t_{j}\right)
$$

which implies $s_{j}>t_{j}$. So $\sum_{j=1}^{e} s_{j}>\sum_{j=1}^{e} t_{j}=1$, which is a contradiction. So $e<f$ is not possible. By symmetry, $e>f$ is also not possible. The result follows.

Finally, we show that $t_{1} \geq t_{2} \ldots \geq t_{d}$. For $1 \leq i<j \leq d^{\prime}$, $M_{i} W\left(t_{i}\right)=M_{j} W\left(t_{j}\right)$ by (24). But $M_{i} \geq M_{j}$ by (20); so $W\left(t_{i}\right) \leq W\left(t_{j}\right)$ and hence, by part (iii) of Lemma $26, t_{i} \geq t_{j}$. For $l>d^{\prime}, t_{l}=0$ by (23). The result follows.

\section{E. Proof of Lemma 4}

In Lemmas 28, 29 and 30 below, we state and prove a generalization of Lemma 4 in which we relax the assumption that $M_{1}, \ldots, M_{d}$ are distinct.

Lemma 28: Let $z=\left|\left\{i: M_{i}=M_{1}\right\}\right|$. If there exists an $\epsilon>0$ such that for all large $n, q<z k_{n} /(n-1)-\epsilon$, then 
$\eta \rightarrow 1, \tilde{p}_{j} \rightarrow \nu, j=1, \ldots, z$ as $n \rightarrow \infty$. Also, for all large $n, d^{\prime}=z, t_{1}=\ldots=t_{z}=1 / z, t_{z+1}=t_{z+2}=\ldots t_{d}=0$.

Proof: Note that for all large enough $n$, for each $i$, $\frac{\sum_{j=1}^{n} q_{j}-q_{i}}{z}<(n-1) q / z+(n-1) \epsilon / 2 z$. Thus, if each primary selects an I.S. w.p. $1 / z$, for a given primary with available bandwidth, the expected number of primaries among the rest minus the expected number of secondaries is less than $-(n-1) \epsilon / 2 z$. Clearly, then, for each $i, w_{i}(1 / z) \rightarrow 0$ as $n \rightarrow \infty$ (convergence is exponentially fast by Hoeffding's inequality [27]). Thus, $W(1 / z) \rightarrow 1$ as $n \rightarrow \infty$. Thus, for all large enough $n, M_{1} W(1 / z)=M_{2} W(1 / z)=$ $\ldots M_{z} W(1 / z)>M_{z+1} r$. Thus, $(1 / z, \ldots, 1 / z, 0, \ldots, 0)$ satisfies the requisite equations for the symmetric NE I.S. selection p.m.f. The last part follows. For $j=1, \ldots, z$, clearly $(\nu-c)\left(1-w_{1}(1 / z)\right) \leq \tilde{p}_{j}-c \leq \nu-c$. Thus, $\tilde{p}_{j} \rightarrow \nu$ as $n \rightarrow \infty$. Thus, the expected utility of any primary with available bandwidth converges to $M_{1}$, the maximum possible value, and the error decays exponentially with increase in $n$. Thus, $\eta \rightarrow 1$.

Lemma 29: Consider $l<d$. Let $l_{\min }=\min \left\{i \leq l: M_{i}=\right.$ $\left.M_{l}\right\}$ and $l_{\max }=\max \left\{i \geq l: M_{i}=M_{l}\right\}$. If there exists an $\epsilon>0$ such that for all large $n, l k_{n} /(n-1)+\epsilon<\bar{q}_{n}<$ $(l+1) k_{n} /(n-1)-\epsilon$, then for all large $n, d_{n}^{\prime} \geq \max (l+$ $\left.1, l_{\max }\right)$. Also, $t_{m n} \bar{q}_{n} \rightarrow k_{n} /(n-1)$ for $m=1, \ldots, l_{\min }-1$ and $t_{m n} \bar{q}_{n} \rightarrow \min \left(\frac{\overline{q_{n}}-\frac{\left(l_{\min }-1\right) k_{n}}{n-1}}{l_{\max }-l_{\min }+1}, k_{n} /(n-1)\right)$ for $m=$ $l_{\text {min }}, \ldots, l_{\text {max }}$.

Proof: First let $d_{n}^{\prime} \leq l$. Then $t_{1 n} \geq 1 / d_{n}^{\prime} \geq 1 / l$. Thus, $t_{1 n} q_{n} \geq k_{n} /(n-1)+\epsilon / l$. Thus, $W\left(t_{1 n}\right) \rightarrow 0$ and $M_{1} W\left(t_{1 n}\right) \rightarrow 0$ as $n \rightarrow \infty$. Thus, $M_{1} W\left(t_{1 n}\right)<M_{l+1}$ for all large enough $n$ (contradiction). Thus, $d_{n}^{\prime} \geq l+1$. However, the fact that $d_{n}^{\prime} \geq l$ implies that $d_{n}^{\prime} \geq l_{\max }$. To prove this, suppose not. Then $M_{l} W\left(t_{l}\right)>M_{l_{\max }} r=M_{l} r$. So $W\left(t_{l}\right)>r$, which contradicts Lemma 26. So $d_{n}^{\prime} \geq l_{\max }$ and hence $d_{n}^{\prime} \geq \max \left(l_{\max }, l+1\right)$. Thus, the first part of the lemma holds.

Now, consider a $m \leq l_{\max }$. Let there exist a $\delta>0$ such that $t_{m n} \bar{q}_{n}>k_{n} /(n-1)+\delta$ for a certain subsequence $\left\{\bar{q}_{n}, k_{n}\right\}$. Then $W\left(t_{m n}\right) \rightarrow 0$ for that subsequence. Thus, $M_{m} W\left(t_{m n}\right) \rightarrow 0$ for that subsequence. Let $d_{n}^{\prime}=d$ in a subsequence of the above subsequence. In this subsequence $t_{d_{n}^{\prime}} \leq 1 / d$, and thus $t_{d_{n}^{\prime}} q_{n}<k_{n} /(n-1)-\epsilon, W\left(t_{d_{n}^{\prime}}\right) \rightarrow 1$ and $M_{d_{n}^{\prime}} W\left(t_{d_{n}^{\prime}}\right)>0$. Thus, $M_{m} W\left(t_{m n}\right) \neq M_{d^{\prime}} W\left(t_{d^{\prime} n}\right)$ for all large enough $n$ (contradiction). Thus, $d_{n}^{\prime}<d$ throughout the above subsequence. But then $M_{m} W\left(t_{m n}\right)<M_{d_{n}^{\prime}+1}$ for all large enough $n$ (contradiction). Thus, no such subsequence exists. Thus, $\lim \sup t_{m n} \leq k_{n} /(n-1)$.

Now, for $m \in\left\{1, \ldots, l_{\text {min }}-1\right\}$, let there exist a $\delta>0$ such that $t_{m n} q_{n}<k_{n} /(n-1)-\delta$ for a certain subsequence $\left\{q_{n}, k_{n}\right\}$. Then $W\left(t_{m n}\right) \rightarrow 1$ for that subsequence. Thus, in that subsequence, $M_{m} W\left(t_{m n}\right)>M_{m+1}$ for all large enough $n$. Then for all large enough $n, d_{n}^{\prime}=m \leq l$ (contradiction). Thus, $\liminf t_{m n} q_{n} \geq k_{n} /(n-1)$. Hence,

$$
t_{m n} q_{n} \rightarrow k_{n} /(n-1), m=1, \ldots, l_{m i n}-1 .
$$

Now, let $m \in\left\{l_{\min }, \ldots, l_{\max }\right\}$. Since $M_{l_{\min }}=\ldots=$ $M_{l_{\max }}$ and $M_{l_{\min }} W\left(t_{l_{\min }}\right)=\ldots=M_{l_{\max }} W\left(t_{l_{\max }}\right)$, it follows that $t_{l_{\min }}=\ldots=t_{l_{\max }}=t_{l}$. Suppose for a subsequence, $t_{l} \bar{q}_{n}>\frac{\bar{q}_{n}-\frac{\left(l_{\min }-1\right) k_{n}}{n-1}}{l_{\max }-l_{\min }+1}+\delta$. This implies

$$
\left(l_{\max }-l_{\min }+1\right) t_{l}+\left(\frac{1}{\bar{q}_{n}} \frac{\left(l_{\min }-1\right) k_{n}}{n-1}\right)>\frac{\delta}{\bar{q}_{n}}+1
$$

Taking limits as $n \rightarrow \infty$ on both sides and using (117) and the fact that $t_{l_{\min }}=\ldots=t_{l_{\max }}=t_{l}$, we get:

$$
\sum_{m=l_{\min }}^{l_{\max }} t_{m}+\sum_{m=1}^{l_{\min }-1} t_{m}>1+\frac{\delta}{q}
$$

which contradicts the fact that $\left(t_{1}, \ldots, t_{d}\right)$ is a probability distribution. Hence,

$$
\limsup t_{l} q_{n} \leq \frac{\bar{q}_{n}-\frac{\left(l_{\min }-1\right) k_{n}}{n-1}}{l_{\max }-l_{\min }+1} .
$$

Now, we consider two cases.

Case (i):

$$
\lim _{n \rightarrow \infty} \frac{\bar{q}_{n}-\frac{\left(l_{\min }-1\right) k_{n}}{n-1}}{l_{\max }-l_{\min }+1} \leq \lim _{n \rightarrow \infty} \frac{k_{n}}{n-1} .
$$

Suppose there exists $\delta>0$ such that for a subsequence $t_{l_{n}}$ :

$$
t_{l_{n}} \bar{q}_{n}<\frac{\bar{q}_{n}-\frac{\left(l_{\min }-1\right) k_{n}}{n-1}}{l_{\max }-l_{\min }+1}-\delta
$$

For this subsequence, after accounting for the probability masses put on $I_{1}, \ldots, I_{l_{\max }}$, there is still some left. So $d^{\prime} \geq l_{\max }+1$ for this subsequence. However, by (118) and (119):

$$
t_{l_{n}} \bar{q}_{n}<\frac{k_{n}}{n-1}-\delta
$$

for large enough $n$. So $W\left(t_{l_{n}}\right) \rightarrow 1$ for the subsequence. So in the subsequence, $M_{l} W\left(t_{l_{n}}\right)>M_{l_{\max }+1}$, which contradicts the fact that $d_{n}^{\prime} \geq l_{\max }+1$. Thus,

$$
\liminf t_{l_{n}} \bar{q}_{n} \geq \frac{\bar{q}_{n}-\frac{\left(l_{\min }-1\right) k_{n}}{n-1}}{l_{\max }-l_{\min }+1}
$$

and hence $t_{l_{n}} \bar{q}_{n} \rightarrow \frac{\bar{q}_{n}-\frac{\left(l_{\min }-1\right) k_{n}}{n-1}}{l_{\max }-l_{\min }+1}$.

Case (ii):

$$
\lim _{n \rightarrow \infty} \frac{k_{n}}{n-1}<\lim _{n \rightarrow \infty} \frac{\bar{q}_{n}-\frac{\left(l_{\min }-1\right) k_{n}}{n-1}}{l_{\max }-l_{\min }+1}
$$

Suppose

$$
t_{l_{n}} \bar{q}_{n}<\frac{k_{n}}{n-1}-\delta
$$

for a subsequence. Then

$$
W\left(t_{l_{n}}\right) \rightarrow 1
$$

for that subsequence. Now, by (121) and (122):

$$
t_{l_{n}} q_{n}<\frac{\bar{q}_{n}-\frac{\left(l_{\min }-1\right) k_{n}}{n-1}}{l_{\max }-l_{\min }+1}
$$

for large enough $n$. So similar to Case (i), after accounting for the probability masses put on $I_{1}, \ldots, I_{l_{\max }}$, there is still some left. So

$$
d_{n}^{\prime} \geq l_{\max }+1
$$


But by (123), $M_{l} W\left(t_{l_{n}}\right)>M_{l_{\max }+1}$, which contradicts (124).

Thus, in Case (ii), $t_{l_{n}} q_{n} \rightarrow \frac{k_{n}}{n-1}$.

Hence, in both cases, $t_{m n} \bar{q}_{n} \rightarrow$ $\min \left(\frac{\overline{q_{n}}-\frac{\left(l_{\min }-1\right) k_{n}}{n-1}}{l_{\max }-l_{\min }+1}, k_{n} /(n-1)\right)$ and we are done.

Lemma 30: If there exists an $\epsilon>0$ such that for all large $n, q>k_{n} d /(n-1)+\epsilon, \eta \rightarrow 0$ as $n \rightarrow \infty$. Also, for all large $n, d^{\prime}=d$ and $\tilde{p}_{j} \rightarrow c, j=1, \ldots, d$.

Proof: Clearly, $t_{1} \geq 1 / d$. Thus, $t_{1} q \geq k_{n} /(n-1)+\epsilon / d$. Now, for all large enough $n$, for each $i, \sum_{j=1}^{n} t_{1} q_{j}-t_{1} q_{i}>$ $(n-1) t_{1} q-t_{1}(n-1) \epsilon / 2$. Thus, if a given primary with available bandwidth selects $I_{1}$, then the expected number of other primaries he sees at a node there minus the expected number of secondaries is greater than $(n-1) \epsilon / 2$. Clearly, then for each $i, w_{i}\left(t_{1}\right) \rightarrow 1$ as $n \rightarrow \infty$ (convergence is exponentially fast by Hoeffding's inequality [27]). Thus, $W\left(t_{1}\right) \rightarrow 0$ and $M_{1} W\left(t_{1}\right) \rightarrow 0$ as $n \rightarrow \infty$. Thus, $M_{1} W\left(t_{1}\right)<M_{d}$ for all large enough $n$. Thus, $d^{\prime}=d$. So for $j=1, \ldots, d, M_{j} W\left(t_{j}\right)=M_{1} W\left(t_{j}\right) \rightarrow 0$ as $n \rightarrow \infty$ and hence $\tilde{p}_{j} \rightarrow c$. Thus, the second part of the lemma holds. Since $M_{1} W\left(t_{1}\right) \rightarrow 0$ as $n \rightarrow \infty$, expected utility of each primary approaches 0 , and the approach is exponentially fast. Thus, the overall expected utility of all primaries approach 0 . Clearly, the expected utility attained by OPT is bounded away from 0 . The result follows. 